Document downloaded from:

http://hdl.handle.net/10251/147695

This paper must be cited as:

Dhakshinamoorthy, A.; Asiri, AM.; Alvaro Rodríguez, MM.; García Gómez, H. (2018). Metal organic frameworks as catalysts in solvent-free or ionic liquid assisted conditions. Green Chemistry. 20(1):86-107. https://doi.org/10.1039/C7GC02260C

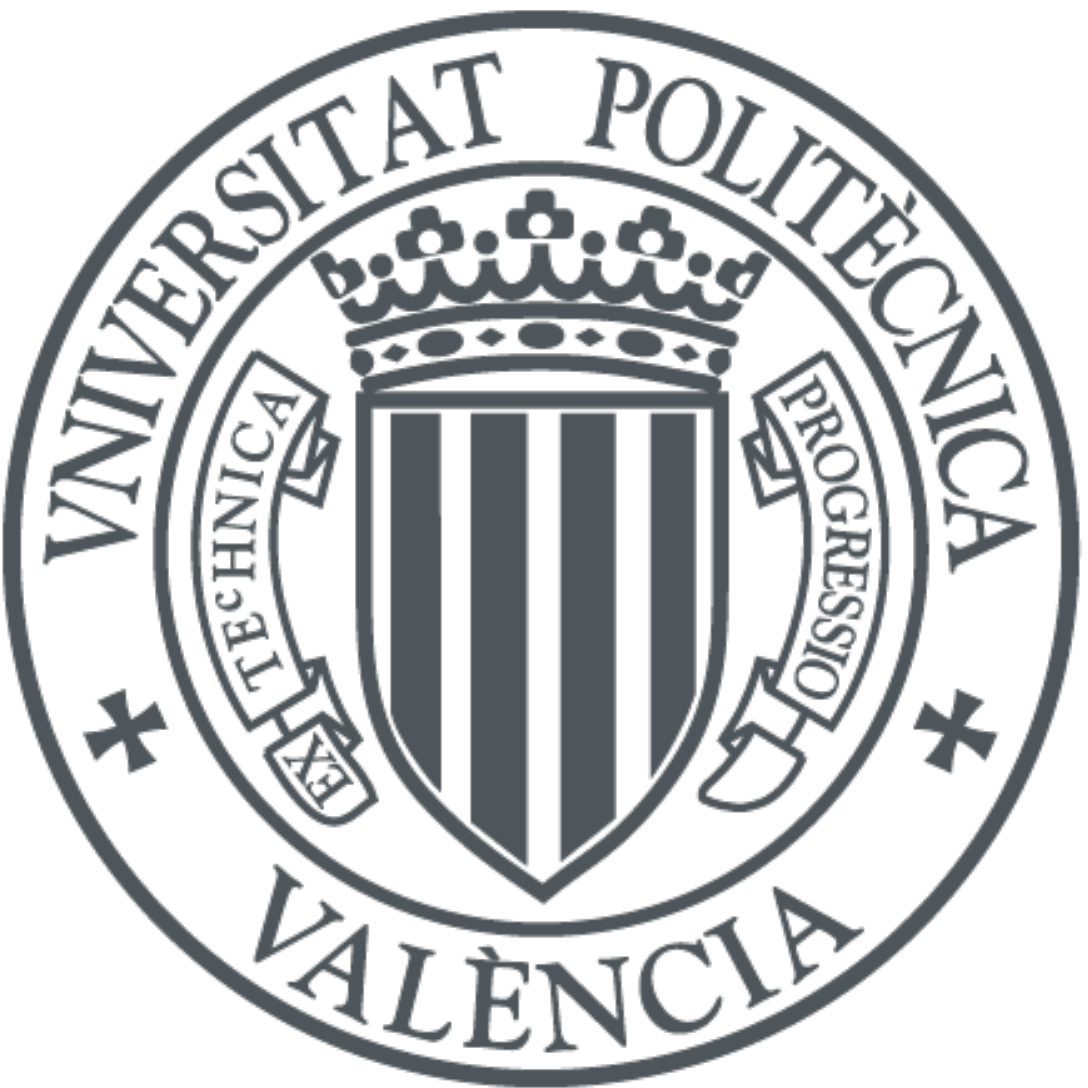

The final publication is available at

https://doi.org/10.1039/C7GC02260C

Copyright The Royal Society of Chemistry

Additional Information 


\title{
Metal Organic Frameworks as Catalysts in Solvent-free or Ionic Liquid Assisted
}

\section{Conditions}

Amarajothi Dhakshinamoorthy, ${ }^{\text {a, b }}$ Abdullah M. Asiri, ${ }^{\mathrm{c}}$ Mercedes Alvaro, ${ }^{\mathrm{b}}$ and Hermenegildo

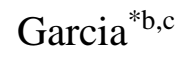

aSchool of Chemistry, Madurai Kamaraj University, Madurai-625 021, Tamil Nadu, India. E-mail: admguru@gmail.com

bepartamento de Quimica and Instituto Universitario de Tecnologia Quimica (CSIC-UPV), Universitat Politecnica de Valencia, Av. De los Naranjos s/n, 46022 Valencia, Spain. E-mail: hgarcia@qim.upv.es

${ }^{\mathrm{c}}$ Center of Excellence in Materials Research, King Abdulaziz University, Jeddah, Saudi Arabia

\begin{abstract}
Metal organic frameworks are being intensively studied as solid catalysts for organic reactions in the liquid media. This review focus on those reports in which these materials have been used as catalysts in the absence of solvents or embedding ionic liquids. One of the major roles of solvents in liquid phase reactions is to desorb reagents and products from the active sites facilitating the turnover of the active sites. For this reason, it is a general observation that most solid catalysts undergo strong deactivation and poisoning in the absence of solvents. In the present review, examples are presented showing that, due to their large porosity and framework flexibility, MOFs can be used as catalysts in the absence of solvent for several reaction types including cyanosilylations, condensations, cyloadditions and $\mathrm{CO}_{2}$ insertions, among others, performing even better than in the presence of conventional organic solvents. It is also described the synergy that arises from the combination of ionic liquids, frequently with suitable task-specific chains, and MOFs due to the cooperation to the catalysis when two centres in MOF and ionic liquid are present and the change in the microenvironment of the active sites.
\end{abstract}


By embedding the ionic liquid in the MOF pores or by synthesising the ionic liquid covalently attached to the ligand in satellite positions, reusable and efficient catalysts can be obtained requiring the minimum amount of ionic liquid. Both complementary strategies increase the greenness of MOF as heterogeneous catalysis and have advantages from the environmental point of view.

\section{Introduction}

Green chemistry is aimed at improving the environmental benignity of chemical processes by reducing the amount of byproducts and wastes formed in a chemical process, by using less toxic and tolerable substrates and reagents and by minimizing as much as possible work-up and energy consumption. ${ }^{1-6}$ To achieve these general goals, one of the most powerful tools is the use of catalysts that should increase the efficiency of chemical reactions and produce selectively the wanted products. ${ }^{6}$ Compared to homogeneous catalysts, heterogeneous catalysts has the advantages of easier recovery of the catalysts from the reaction mixture, the possibility to reuse the catalyst in consecutive runs and their adaptability to continuous flow processes that are better suited for large scale production. Heterogeneous catalysis is mainly based on the use of insoluble solids that should exhibit high surface area to favour the contact of the active sites with substrates and reagents. Among the various types of solid catalysts, one that is currently attracting considerable attention is metal organic frameworks (MOFs). ${ }^{7-9}$

MOFs are porous crystalline materials whose lattice is constituted by metallic nodes coordinated with rigid multipodal organic linkers, mostly aromatic polycarboxylates. ${ }^{10-12}$ The catalytic activity of these materials derives from the presence of coordinatively unsaturated sites (CUS) around the metal ions at the nodes and structural defects, from the presence of acid or basic substituents at the ligands or from the presence of guests species located within the internal voids of the structure. ${ }^{13-15}$ The wide use of MOFs as catalysts is understandable 
considering the large choice in the nature of the metal that can form part of the MOF, and the possibility to adapt the MOF properties after the synthesis. Other suitable features of MOFs as catalysts are their large surface area and high pore volume and the flexibility in the synthesis of these materials. In this way, MOFs have become among the preferred solid catalysts for liquid phase reactions including oxidations, ${ }^{16,17}$ condensations, ${ }^{18}$ couplings, ${ }^{9}$ among others. ${ }^{19}$ The use of MOFs as solid catalysts can contribute to the greenness of the process, but other important factors to be considered regardless the catalyst are the nature of reagents and solvents employed in the reaction. Particularly, one of the main sources of undesirable environmental impact is volatile organic solvents, specially those containing halogens that have shown adverse effect for the ozone layer in the atmosphere as well as for the human health. For this reason, one of the lines in green chemistry is aimed at finding suitable alternatives to conventional low boiling point organic solvents that can be emitted to the atmosphere or can become a waste after the reaction.

One of the simplest approaches to avoid the use of volatile organic solvents is to perform the reaction in the absence of any solvent, just be using substrate/reagents that are liquid under the reaction conditions. While solvent-free conditions are favourable from the point of view of avoiding emissions of vapours to the atmosphere, the absence of solvents generates considerable stress on the catalyst, particularly when using solid porous materials. This is because one of the main roles of solvents is to assist reagent, product or byproduct desorption from the active sites by competing with them. In other words, in the absence of solvents, it is a general observation that solid catalysts become easily deactivated by strong adsorption on the active sites of chemicals, including reagents, products and byproducts.

The use of solvent-free conditions on a solid catalyst is, as previously commented, very convenient from the green chemistry point of view, but, however, not very practical due to the occurrence of fast catalyst deactivation. In the present review, examples will be shown 
illustrating that there are several general reaction types that have proven to be successful under solvent-free conditions in the presence of MOFs. The present state of the art has shown that silylations, condensations including aldolic reactions and heterocycle formation, as well as selective hydrogenations and $\mathrm{CO}_{2}$ insertions can be carried out by MOFs in the absence of organic solvents. Although solventless conditions are not exclusive of MOFs and other classes of catalysts have also been used in the absence of solvents, the purpose is to show that some general reaction types can be carried out advantageously using MOFs as catalysts without solvents.

Besides solvent-less conditions, another alternative to the use of volatile organic compounds that has also become a research front in catalysis is the use of ionic liquids (ILs). ${ }^{20-}$ ${ }^{22}$ They have a negligible vapour pressure and it has been shown that they can dissolve a large variety of organic compounds and can also be a convenient liquid media for organic reactions. One of the main problems of ILs is their viscosity that complicates considerably the extraction of products and chemicals after the reaction as well as the recovery of the catalysts. In this context, one of the strategies that has been developed to take advantage of the benefits of the use of ILs as reaction medium as well as to diminish as much as possible the volume of ILs and simplifying the reaction work-up has been to deposit these ILs as a thin film on the surface of large surface area materials. ${ }^{23}$ The presence of porosity of MOFs makes possible to incorporate ILs inside these solid catalysts and in this way, the advantages of heterogeneous catalysis are combined with the benefits of ILs in terms of cooperation to the reaction mechanism, while still being recoverable together with the solid after the reaction. For these reasons, this review also covers those reports combining MOFs and ILs in minimal amount. Particularly relevant are those examples in which task specific ILs i.e. those ILs that have been designed having functional groups to promote organic reactions while maintaining their solubility properties will also be commented. As it will be shown, the combination of IL that 
have Brönsted acidity/basicity with the active sites of MOFs can serve to preserve the catalytic activity of MOFs in Knoevenagel condensations, $\mathrm{CO}_{2}$ insertions and also to avoid the particle size increase that is a common deactivation mechanism observed when supported metal nanoparticles (NPs) are used as catalysts. In addition, another possibility to increase the catalytic activity of MOFs is the use of hierarchical structures in which reagent and substrate diffusion is facilitated by the presence of meso- and micropores.

The aim of the present review is to show the current state of the art of the use of MOFs as catalysts in the absence of solvents, incorporating ILs within the pores in such a way that they form a system with the MOF or exhibiting a hierarchical structure with the presence of meso and micropores. While there is abundant literature reporting the use of MOFs as catalysts in the presence of solvents, this review aims at demonstrating that MOF can even perform with high activity and selectivity in the absence of solvents without compromising their stability, thus fulfilling one of the principles of “Green Chemistry”. On the other hand, the present review also shows the utility of IL attached to MOFs to provide a microenvironment to the active sites or to provide additional sites to enhance the activity of these materials compared to the activity of parent MOFs. A final aspect included in the present review is hierarchical structuration to facilitate accessibility of substrates and reagents to the active sites.

The following Table 1 lists the reactions and the MOF catalysts that will be included in the present review. Emphasis is made on the evidence provided on the stability of the MOF structure under reaction conditions.

Table 1. List of MOFs and reactions that have been reported under solvent-less conditions and stability data.

\begin{tabular}{|l|l|l|l|}
\hline Catalyst & Reaction & $\begin{array}{l}\text { Stability } \\
\text { evidences }\end{array}$ & Ref. \\
\hline
\end{tabular}




\begin{tabular}{|c|c|c|c|}
\hline $\begin{array}{l}\text { vanadium-salen based Cd- } \\
\text { MOF }\end{array}$ & \multirow{7}{*}{$\begin{array}{l}\text { Cyanosilylation of } \\
\text { benzaldehyde }\end{array}$} & XRD, UV-Vis. & 24 \\
\hline In-PF-6 & & XRD, reuse & 25 \\
\hline$\left[\mathrm{In}_{4}(\mathrm{OH})_{4}\left(\mathrm{hfipbb}_{4}\left(4,4^{\prime}\right.\right.\right.$-bipy $\left.)\right]$ & & $\begin{array}{l}\text { Reuse, hot- } \\
\text { filtration }\end{array}$ & 26 \\
\hline$\left[\left(\mathrm{Cu}_{4} \mathrm{O}_{0.27} \mathrm{Cl}_{0.73}\right)_{3}\left(\mathrm{H}_{0.5} \mathrm{BTT}\right)_{8}\right]$ & & XRD, reuse & 27 \\
\hline $\begin{array}{l}\text { [Nd(3,5- } \\
\text { DSB)(Phen)] (RPF-19) }\end{array}$ & & XRD, reuse & 28 \\
\hline$\left[\mathrm{Cd}_{3}(\mathrm{tipp})(\mathrm{bpdc})_{2}\right] \cdot \mathrm{DMA} \cdot 9 \mathrm{H}_{2} \mathrm{O}$ & & $\begin{array}{l}\text { Leaching, } \\
\text { reuse }\end{array}$ & 29 \\
\hline $\mathrm{Ba}_{2}\left(\mathrm{H}_{2} \mathrm{LOMe}\right)_{2}(\mathrm{NMP}) . \mathrm{NMP}$ & & reuse & 30 \\
\hline $\begin{array}{l}\mathrm{M}\left(4,4^{\prime} \text {-bpe }\right)_{2}\left(\mathrm{H}_{2} \mathrm{O}\right)_{4}(m-\mathrm{bds}) \\
\left(\mathrm{M}: \mathrm{Zn}^{2+} \text { or } \mathrm{Cd}^{2+}\right.\end{array}$ & $\begin{array}{l}\text { synthesising 2-amino-6- } \\
\text { (arylthio)pyridine-3,5- } \\
\text { dicarbonitriles }\end{array}$ & reuse & 31 \\
\hline IRMOF-3 & $\begin{array}{lr}\text { Synthesis } & \text { of } \\
\text { dihydropyrimidinones } & \text { and } \\
\text { dihydropyridines } & \end{array}$ & - & 32 \\
\hline IRMOF-3 & $\begin{array}{l}\text { synthesis of } \\
\text { polyhydroquinoline }\end{array}$ & - & 33 \\
\hline PTA@MIL-101 & $\begin{array}{l}\text { Synthesis of 3,4- } \\
\text { dihydropyrimidin-2(1H)-one }\end{array}$ & $\begin{array}{l}\text { Hot-filtration, } \\
\text { reuse, FT-IR, } \\
\text { FESEM }\end{array}$ & 34 \\
\hline$[\mathrm{TPA}]_{2}\left[\mathrm{Zn}_{2}-(\mathrm{BDC})_{3}(\mathrm{DMA})_{2}\right]$ & $\begin{array}{l}\text { Michael addition of pyrrole } \\
\text { to } \beta \text {-nitrostyrenes }\end{array}$ & XRD, reuse & 35 \\
\hline $\mathrm{Cu}_{3}(\mathrm{BTC})_{2}$ & $\begin{array}{l}\text { Michael addition between } \beta \text { - } \\
\text { nitrostyrene and indole }\end{array}$ & - & 36 \\
\hline$[\mathrm{TPA}]_{2}\left[\mathrm{Zn}_{2}-(\mathrm{BDC})_{3}(\mathrm{DMA})_{2}\right]$ & $\begin{array}{lr}\text { Knoevenagel } & \begin{array}{r}\text { condensation } \\
\text { reaction }\end{array} \\
\text { benzaldehyde } & \text { between } \\
\text { malononitrile } & \text { and }\end{array}$ & XRD, reuse & 37 \\
\hline $\mathrm{Cu}(\mathrm{ptz})(\mathrm{nsa})_{0.5 \cdot \mathrm{H}_{2} \mathrm{O}}$ & azide-alkyne cycloaddition & XRD, reuse & 38 \\
\hline $\mathrm{Cu}(\mathrm{I})-\mathrm{MOF}$ & Synthesis of propargylamine & $\begin{array}{l}\text { XRD, reuse, } \\
\text { leaching }\end{array}$ & 39 \\
\hline MIL-101(Cr) & $\begin{array}{l}\text { coupling of } \mathrm{CO}_{2} \text { with styrene } \\
\text { oxide }\end{array}$ & Reuse, XRD & 40 \\
\hline F-IRMOF-3 & coupling of $\mathrm{CO}_{2}$ and & XRD & 41 \\
\hline$\left[\mathrm{Zn}_{4} \mathrm{O}(2,6-\mathrm{NDC})(\mathrm{BTB})_{4 / 3}\right]$ & propylene oxide & XRD, reuse & 42 \\
\hline ABIL-OH/HKUST-1 & $\begin{array}{l}\text { Knoevenagel condensation } \\
\text { between benzaldehyde and } \\
\text { malononitrile }\end{array}$ & $\begin{array}{l}\text { XRD, hot- } \\
\text { filtration }\end{array}$ & 43 \\
\hline $\mathrm{PdCl}_{2}-\mathrm{ILs} / \mathrm{Cu}_{3}(\mathrm{BTC})_{2}$ & oxidation of cyclohexene & $\begin{array}{l}\text { Reuse, XPS, } \\
\text { XRD }\end{array}$ & 44 \\
\hline IL@UiO-66 & oxidative desulfurization & reuse & 45 \\
\hline
\end{tabular}




\begin{tabular}{|c|c|c|c|}
\hline PW/DAIL/MIL-101(Cr) & oxidation of benzyl alcohol & $\begin{array}{l}\text { XRD, reuse, } \\
\text { leaching }\end{array}$ & 46 \\
\hline $\mathrm{PW}_{4} / \mathrm{DAIL} / \mathrm{MIL}-100(\mathrm{Fe})$ & oxidation of benzyl alcohol & $\begin{array}{l}\text { XRD, reuse, } \\
\text { leaching }\end{array}$ & 47 \\
\hline $\begin{array}{l}\mathrm{DAIL}-\mathrm{Fe}_{3} \mathrm{O}_{4} @ \mathrm{NH}_{2}-\mathrm{MIL}- \\
88 \mathrm{~B}(\mathrm{Fe})\end{array}$ & \multirow{2}{*}{$\begin{array}{l}\text { esterification of oleic acid } \\
\text { with ethanol }\end{array}$} & reuse & 48 \\
\hline $\begin{array}{l}{\left[\mathrm{SO}_{3} \mathrm{H}-\left(\mathrm{CH}_{2}\right)_{3-}\right.} \\
\mathrm{HIM}]_{3} \mathrm{PW}_{12} \mathrm{O}_{40} @ \mathrm{MIL}-100(\mathrm{Fe})\end{array}$ & & reuse & 49 \\
\hline $\begin{array}{l}\text { [NMP] }{ }^{+} \mathrm{CH}_{3} \mathrm{SO}_{3}{ }^{-} @ \mathrm{MIL}- \\
101(\mathrm{Cr})\end{array}$ & $\begin{array}{l}\text { Esterification of acetic acid } \\
\text { with amyl alcohol }\end{array}$ & $\begin{array}{l}\text { Reuse, } \\
\text { leaching, TEM }\end{array}$ & 50 \\
\hline TEDA-BAIL/MIL-101 & $\begin{array}{l}\text { Acetalization of } \\
\text { benzaldehyde with glycerol }\end{array}$ & $\begin{array}{l}\text { Reuse, } \\
\text { leaching }\end{array}$ & 51 \\
\hline $\mathrm{Pd} / \mathrm{IL} / \mathrm{Cu}_{3}(\mathrm{BTC})_{2}$ & Hydrogenation of acetylene & $\begin{array}{l}\text { Reuse, XRD, } \\
\text { TEM }\end{array}$ & 52 \\
\hline $\mathrm{Cu}-\mathrm{MOF}$ & $\begin{array}{l}\text { hydrocarboxylation } \\
\text { cyclohexane }\end{array}$ & $\begin{array}{l}\mathrm{XRD} \text {, leaching, } \\
\text { reuse }\end{array}$ & 53 \\
\hline CuI@UiO-67-IM & azide-alkyne cycloaddition & $\begin{array}{l}\text { Reuse, } \\
\text { leaching, XPS }\end{array}$ & 54 \\
\hline $\begin{array}{l}\text { MIL- } 101(\mathrm{Cr})-\mathrm{X}(\mathrm{n}-\mathrm{Bu})_{3} \mathrm{Br}(\mathrm{X}: \\
\mathrm{N} \text { or } \mathrm{P})\end{array}$ & $\begin{array}{l}\text { Coupling of } \mathrm{CO}_{2} \quad \text { and } \\
\text { propylene oxide }\end{array}$ & $\begin{array}{ll}\text { ICP, } & \text { FT-IR, } \\
\text { XRD } & \\
\end{array}$ & 55 \\
\hline UiO-67-IL & $\begin{array}{llll}\text { Coupling of } & \mathrm{CO}_{2} & \text { to } \\
\text { epichlorohydrin } & & \\
\end{array}$ & XRD, leaching & 56 \\
\hline IL-ZIF-90 & $\begin{array}{l}\text { Coupling of } \mathrm{CO}_{2} \quad \text { with } \\
\text { propylene oxide }\end{array}$ & $\begin{array}{l}\text { Reuse, XRD, } \\
\text { leaching }\end{array}$ & 57 \\
\hline
\end{tabular}

InPF: indium polymeric framework; hfipbb: hexafluoroisopropylidene bisbenzoate; bipy: bipyridine; BTT: 1,3,5-benzene tristetrazolate; RPF: rare earth polymer framework; DSB: 3,5disulfobenzoate; Phen: 1,10-phenathroline; tipp $=$ 5,10,15,20-tetrakis(4-(imidazol-1yl)phenyl)-porphyrin; bpdc: biphenyl-4,4'-dicarboxylic acid; DMA: N,N'-dimethylacetamide; H2LOMe: 5,5'-(2,3,6,7-tetramethoxyanthracene-9,10-diyl)diisophthalic acid; NMP： Nmethylpyrrolidine; 4,4'-bpe = 1,2-bis(4-pyridyl)ethylene; $m$-bds = 1,3-benzenedisulfonic acid; PTA: phosphotungstic acid; TPA: tetrapropylammonium; BDC: 1,4-benzenedicarboxylate; DMA: dimethylamine; BTC: 1,3,5-benzenetricarboxylate; ptz: 2,4,6-tri-(4-pyridyl)-1,3,5triazine; nsa: sodium 2,6-naphthalene disulfonate; BTB: 1,3,5-tris(4-carboxyphenyl)benzene; NDC: naphthalenedicarboxylic acid; ABIL: amino-functionalized basic ionic liquid; DAIL: dual amino-functionalized ionic liquid. 


\section{MOFs as Catalysts under Solvent-Free Conditions}

\subsection{Addition of trimethylsilyl cyanide (TMSCN) to carbonyl compounds}

Nucleophilic additions to carbonylic compounds are general organic reactions with a large versatility for the preparation of alcohol derivatives. Cyanohydrins can be easily obtained by cyanide addition to carbonyl groups. In this regard, TMSCN is one of the most useful and safe reagents for nucleophilic addition of cyanide to carbonyl compounds to give the corresponding cyanohydrin trimethylsilyl ethers. Hence, the development of efficient catalysts for cyanosilylation of carbonyl compounds by TMSCN is an important subject in the context of the preparation of cyanohydrins. Several efficient Lewis acid catalysts have been developed so far for the cyanosilylation. ${ }^{58-62}$ Solvent-free conditions can be suitable for the synthesis of various chiral products with interest in biomedicinal chemistry such as $\alpha$-hydroxy acids, $\alpha$ hydroxy aldehydes and $\beta$-amino alcohols ${ }^{61,63}$ through the corresponding cyanohydrin.

Recently, the synthesis of a chiral vanadium-salen based Cd-MOF with a chiral ligand (R,R)-(-)-1,2-cyclohexanediamino-N,N'-bis(3-tert-butyl-5-(4-pyridyl)salicylidene) has been reported under solvothermal conditions and its catalytic activity has been examined in the solvent-free cyanosilylation of benzaldehyde (Scheme 1). ${ }^{24}$ Chiral VO-salen complexes were used as homogeneous asymmetric catalysts for various types of organic reactions. ${ }^{64,65}$ Under optimized conditions, the reaction of benzaldehyde with TMSCN catalyzed by chiral VO-salen attached to Cd-MOF gave 95\% yield with 78\% ee. In contrast, the same reaction afforded 91\% yield with 57\% ee using VO-salen as homogeneous catalyst. These data clearly indicate the accessibility of substrates to the chiral centers located inside the pores of Cd-MOF. On the other hand, the observed activity with this Cd-MOF under solvent-less conditions is comparable with other related chiral MOFs in the presence of solvents. ${ }^{66,67}$ Furthermore, control experiments performed in the presence of chloroform and acetonitrile detected the 
occurrence of leaching, thus showing the convenience of carrying out organic reactions under solvent-free conditions, although the exact reasons why leaching does not occur in the absence of solvents remain unknown. The activity of this catalyst was maintained in two runs, although a decrease in the yield and ee was noticed in the third run. This deterioration of the catalytic activity was believed to be due to the modification of the surrounding environment of chiral centers or/and undesirable adsorption of organic compounds, since. However, in spite of the decrease in activity, the catalyst maintained its crystallinity after the third run and the vanadium oxidation state did not show remarkable changes.

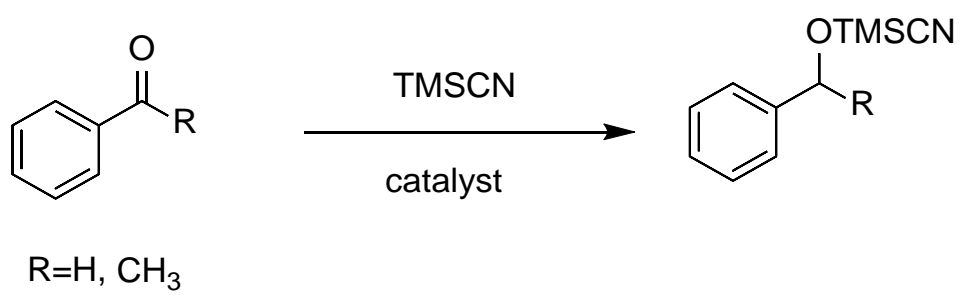

Scheme 1. Cyanosilylation of benzaldehyde/acetophenone with TMSCN.

The use of a new flexible ligand in the synthesis of two In-MOFs with ancillary coordination resulted in one one-dimensional and two isoreticular 2D structures, their catalytic activity being evaluated in the cyanosilylation of carbonyl compounds. ${ }^{25}$ It was found that those In MOFs with donor ancillary ligands in their structures show the best activity, the one having 1,10-phenanthroline being the most active. The turnover frequency (TOF) value reached in the cyanosilylation of benzaldehyde with the most active In-MOF was $2149 \mathrm{~h}^{-1}$ with $99 \%$ yield at $50{ }^{\circ} \mathrm{C}$ under solvent-free conditions. Furthermore, the cyanosilylation of acetophenone at 80 ${ }^{\circ} \mathrm{C}$ with the best-performing In-MOF exhibited $39 \%$ yield with a TOF value of $19 \mathrm{~h}^{-1}$. The recyclability data indicated that the In-MOF could be reused at least four times, maintaining according to powder XRD its crystallinity, but exhibiting, however, a small loss in its catalytic activity.

Later, four new indium MOFs, namely $\left[\operatorname{In}_{2}(\text { hfipbb) })_{3}(1,10-p h e n)_{2}\right] .2 \mathrm{H}_{2} \mathrm{O}$ (phen: phenantroline) (InPF-12), [ $\operatorname{In}_{2}$ (hfipbb) $\left.)_{3}\left(2,2^{\prime} \text {-bipy }\right)_{2}\right] .2 \mathrm{H}_{2} \mathrm{O}$ (InPF-13), [ $\operatorname{In}_{2}(\text { hfipbb) })_{3}(4,4$ '-bipy)] 
(InPF-14) and [ $\mathrm{In}_{4}(\mathrm{OH})_{4}(\text { hfipbb) })_{4}\left(4,4^{\prime}\right.$-bipy) $]$ (InPF-15), were hydrothermally synthesised and their activity in the solvent-free cyanosilylation of carbonyl compounds also studied. ${ }^{26}$ Among these catalysts, In-PF-15 exhibited better activity in cyanosilylation of benzaldehyde and acetophenone compared to other analogous catalysts (Table 2). As an example, the cyanosilylation of cyclohexanone using TMSCN in the presence of In-PF-15 resulted in a TOF value of $12048 \mathrm{~h}^{-1}$ at $80{ }^{\circ} \mathrm{C}$ with 0.1 mol\% catalyst loading under nitrogen atmosphere. InPF15-maintains its crystallinity after seven catalytic cycles and hot filtration experiments supported the heterogeneity of the process.

Table 2. Activity data of In(III)-MOFs as catalysts for cyanosilylation reaction. ${ }^{\text {a }}$ Data adapted from ref. ${ }^{26}$

\begin{tabular}{|l|l|l|l|l|}
\hline \multirow{2}{*}{ Catalyst } & \multicolumn{3}{|l|}{ Benzaldehyde } & \multicolumn{2}{l|}{ Acetophenone $^{\mathrm{c}}$} \\
\cline { 2 - 5 } & Yield $(\%),(\mathrm{h})$ & $\begin{array}{l}\mathrm{TOF}^{\mathrm{d}} \\
\left(\mathrm{h}^{-1}\right)\end{array}$ & Yield $^{\mathrm{b}}(\%),(\mathrm{h})$ & $\begin{array}{l}\mathrm{TOF}^{\mathrm{d}} \\
\left(\mathrm{h}^{-1}\right)\end{array}$ \\
\hline InPF-11 & $99(0.67)$ & 105 & $65(4)$ & 109 \\
\hline InPF-12 & $99(2)$ & 284 & $24(4)$ & 1 \\
\hline InPF-13 & $93(2)$ & 298 & $41(2)$ & 38 \\
\hline InPF-14 & $99(0.75)$ & 332 & $45(1)$ & 50 \\
\hline InPF-15 & $80(1.5)$ & 265 & $77(4)$ & 140 \\
\hline
\end{tabular}

${ }^{a}$ Without solvent, $2.5 \mathrm{~mol} \%$ of catalyst under $\mathrm{N}_{2}$ atmosphere, $50{ }^{\circ} \mathrm{C}$ (benzaldehyde) or $80{ }^{\circ} \mathrm{C}$ (acetophenone). ${ }^{\mathrm{b}}$ Based on GC-MS. ${ }^{\mathrm{c}}$ All reactions were completed at $7 \mathrm{~h} .{ }^{\mathrm{d}} \mathrm{TOF}$ : mmol substrate/mmol catalyst $\mathrm{x}$ h.

Recently, solvothermal reaction between $\mathrm{Cu}(\mathrm{OAc})_{2}$ and $\mathrm{H}_{3} \mathrm{BTT} .2 \mathrm{HCl}$ produced a sodalite-type MOF with a structural formula of $\left[\left(\mathrm{Cu}_{4} \mathrm{O}_{0.27} \mathrm{Cl}_{0.73}\right)_{3}\left(\mathrm{H}_{0.5} \mathrm{BTT}\right)_{8}\left(\mathrm{H}_{2} \mathrm{O}\right)_{12}\right] .3 \mathrm{MeOH} .9 \mathrm{DMF} .{ }^{27}$ This MOF exhibits a porous 3D $(3,8)-$ connected framework constructed by square $\left[\mathrm{Cu}_{4}\left(\mu_{4}-\mathrm{O} / \mathrm{Cl}\right)\right]$ units and triangular BTT ligands. Even further, this material can be dehydrated removing solvent molecules to form $\left[\left(\mathrm{Cu}_{4} \mathrm{O}_{0.27} \mathrm{Cl}_{0.73}\right)_{3}\left(\mathrm{H}_{0.5} \mathrm{BTT}\right)_{8}\right]$ possessing coordinatively unsaturated $\mathrm{Cu}^{2+}$ centres. Interestingly, the activity of $\left[\left(\mathrm{Cu}_{4} \mathrm{O}_{0.27} \mathrm{Cl}_{0.73}\right)_{3}\left(\mathrm{H}_{0.5} \mathrm{BTT}\right)_{8}\right]$ at $1 \mathrm{~mol} \%$ for cyanosilylation of benzaldehyde led to a $96 \%$ conversion of benzaldehyde at $40{ }^{\circ} \mathrm{C}$ under a $\mathrm{N}_{2}$ atmosphere, which 
is one eleventh of the loading used in related Mn-BTT catalyst. ${ }^{68}$ On the other hand, the reactivity of $\left[\left(\mathrm{Cu}_{4} \mathrm{O}_{0.27} \mathrm{Cl}_{0.73}\right)_{3}\left(\mathrm{H}_{0.5} \mathrm{BTT}\right)_{8}\right]$ was higher than that of Sc-MOF and Ln-MOFs in which the loading of catalysts was 5 mol\%. ${ }^{28,69}$ Leaching tests under optimized reaction conditions demonstrated the heterogeneity of the process. Notably, the use of 1-naphthaldehyde as substrate gave only $78 \%$ of product, while the use of 9-anthracenecarboxaldehyde did not afford any conversion. These experimental data are in clear agreement with the expectations of size selective catalysis, implying that the cyanosilylation occurs within the pores of $\left[\left(\mathrm{Cu}_{4} \mathrm{O}_{0.27} \mathrm{Cl}_{0.73}\right)_{3}\left(\mathrm{H}_{0.5} \mathrm{BTT}\right)_{8}\right]$. The catalyst could be reused five times for the reaction of furfuraldehyde and TMSCN without noticeable loss of activity.

Three Ln-based 2D MOFs with the formula [Ln(3,5DSB $)($ Phen $)](\mathrm{Ln}=\mathrm{La}, \operatorname{Pr}(\mathrm{RPF}-18) ; \mathrm{Ln}=\mathrm{Nd}(\mathrm{RPF}-19)$ were hydrothermally synthesized and their activity in the cyanosilylation of carbonyl compounds examined ${ }^{28}$ RPF-19-Nd exhibits the highest activity for the cyanosilylation of benzaldehyde with 5 mol\% catalyst loading at $50{ }^{\circ} \mathrm{C}$ under $\mathrm{N}_{2}$ atmosphere exhibiting a TOF value of $12.94 \mathrm{~h}^{-1}$. Furthermore, the reactivity of RPF-19-Nd (benzaldehyde, 2 h, 94.8 \% yield) was higher than that of Mn-MOF (benzaldehyde, $9 \mathrm{~h}, 98 \%$ yield), ${ }^{68}$ the latter requiring solvent. In contrast, the activity of RPF-19-Nd was slightly lower than Ln-MOF (benzaldehyde, 1 h, $88 \%$ yield), ${ }^{70}$ but however, the latter requires a pre-activation treatment. The RPF-19-Nd catalyst was reused for four consecutive runs; without observing. The synthesis of a porous 4-fold interpenetrated MOF, namely, [Cd 3 (tipp)(bpdc) 2 · DMA $9 \mathrm{H}_{2} \mathrm{O}$ has been reported and its activity tested for the solvent-free cyanosilylation of carbonyl compounds. ${ }^{29}$ The reaction of benzaldehyde with TMSCN afforded $94 \%$ yield of the desired product with $6 \mathrm{~mol} \%$ of $\left[\mathrm{Cd}_{3}(\mathrm{tipp})(\mathrm{bpdc})_{2}\right] \cdot \mathrm{DMA} \cdot 9 \mathrm{H}_{2} \mathrm{O}$ at room temperature with a turnover number (TON) value of 156.7. Moreover, no catalytic activity was observed for acetophenone and 4-methylacetophenone in the cyanosilylation reactions with $\left[\mathrm{Cd}_{3}(\right.$ tipp)(bpdc) 2$] \cdot \mathrm{DMA} \cdot 9 \mathrm{H}_{2} \mathrm{O}$. 
The solvothermal reaction of 5,5'-(2,3,6,7-tetramethoxyanthracene-9,10diyl)diisophthalic acid $\left(\mathrm{H}_{4} \mathrm{~L}\right.$, Scheme 2) and $\mathrm{Ba}\left(\mathrm{NO}_{3}\right)_{2}$ resulted in the formation of a microporous MOF, namely, $\mathrm{Ba}_{2}\left(\mathrm{H}_{2} \mathrm{LOMe}\right)_{2}(\mathrm{NMP})$.NMP with high thermal stability. Upon heating at $325{ }^{\circ} \mathrm{C}, \mathrm{Ba}_{2}\left(\mathrm{H}_{2} \mathrm{LOMe}\right)_{2}(\mathrm{NMP})$. NMP transformed to $\left[\mathrm{Ba}\left(\mathrm{H}_{2} \mathrm{LOMe}\right)_{0.5}\left(\mathrm{H}_{2} \mathrm{O}\right) .4 \mathrm{H}_{2} \mathrm{O}\right]_{\mathrm{n}}$, through a single-crystal-to-single-crystal conversion. ${ }^{30}$ Furthermore, the formation of an intermediate was observed and ts catalytic activity studied in the cyanosilylation of carbonyl compounds with TMSCN. The intermediate Ba MOF catalyst showed $100 \%$ yields for acetophenone, $p$-methylacetophenone, $p$-chloroacetophenone, $p$-nitroacetophenone, and 4phenylbut-3-en-2-one at room temperature. Moreover, the TON and TOF for the cyanosilylation of benzaldehyde was 192 and $384 \mathrm{~h}^{-1}$, respectively, which are much higher than those achieved for other MOF catalysts commented above. Also, the activity of this intermediate catalyst was higher than for polymer-supported metal complexes and $\mathrm{SbCl}_{3}$-based catalysts. $^{71,72}$ The Ba MOF catalyst maintained its activity in the cyanosilylation of benzaldehyde in three consecutive cycles.<smiles>COc1cc(C(=O)O)cc(-c2c3cc(OC)c(OC)cc3c(-c3cc(C(=O)O)cc(C(=O)O)c3)c3cc(OC)c(OC)cc23)c1</smiles>

Scheme 2. Structure of the ligand $\mathrm{H}_{4} \mathrm{~L}$.

\subsection{Heterocycle Synthesis}

Condensation reactions of carbonylic compounds are widely used for the synthesis of six and five member heterocycles, including N-containing ones with pyridine, dihydropyridine 
or the substituted analogues. MOFs have been used as catalysts for the synthesis of these compounds under solvent-free conditions.

The activity of $\mathrm{M}\left(4,4^{\prime} \text {-bpe }\right)_{2}\left(\mathrm{H}_{2} \mathrm{O}\right)_{4}(m$-bds $)\left(\mathrm{M}=\mathrm{Zn}^{2+}\right.$ or $\left.\mathrm{Cd}^{2+}\right)$ was investigated in the synthesis of 2-amino-6-(arylthio)pyridine-3,5-dicarbonitriles under solvent-free conditions using benzaldehyde, malononitrile, and thiophenol as reagents as shown in Scheme $3 .{ }^{31}$ The use of Zn and Cd MOFs as catalysts gave 86 and $87 \%$ yield, respectively, under solvent-free conditions, while the same MOFs required twenty times longer time to achieve comparable yields using toluene under identical reaction conditions. These experiments clearly demonstrate the advantage of performing organic transformations under solvent-free conditions by reducing the time as well as energy consumption associated in the process. It could be that the excellent packing already documented for alkyl aromatics inside the cavities of certain MOFs, disfavors the accessibility for substrates inside MOFs, ${ }^{73}, 74$ just the opposite role that the solvents are supposed to play. Furthermore, these catalysts also afforded the desired product with substituted thiophenols in high yields (81-87 \%) under solvent-free conditions.

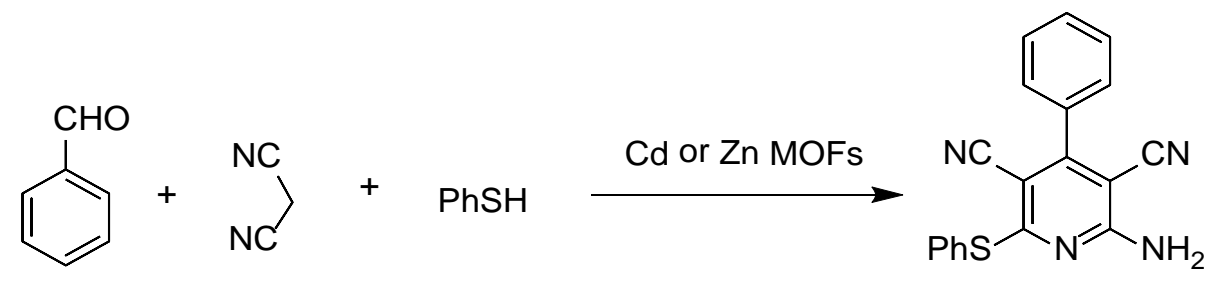

Scheme 3. Synthesis of pyridine derivatives using Cd or Zn MOFs.

Dihydropyrimidinones (DHPMs) exhibit a variety of important biological activities, including calcium channel blockers, antihypertensives and adrenergic antagonists. On the other hand, dihydropyridines (DHPs) are among the most widely used drugs, some relevant examples being shown in Scheme 4. These heterocyclic rings are also known as neuroprotectants because they exhibit anti-platelet aggregation properties and, therefore, they are important in Alzheimer's disease treatments and as anti-ischemic agents. ${ }^{75-78}$ 
<smiles>CCOC(=O)/C=C\c1ccccc1C1C(C(=O)OCC)=C(C)NC(C)=C1C(=O)OCC</smiles>

Lacidipine<smiles>COC(=O)C1=C(C)NC(C)=C(C(=O)OC)C1c1ccccc1[N+](=O)[O-]</smiles>

Nifedipine<smiles>CC1=C(C(=O)OC(C)C)C(c2cccc([N+](=O)[O-])c2)N(C(N)=O)C(=O)N1</smiles>

SQ32926

Scheme 4. Examples of biologically important DHPM and DHP derivatives.

Most of these nitrogen heterocycles are synthesised using a large variety of homogeneous catalysts. In this context, isoreticular MOFs (IRMOF-3) has been reported as heterogeneous catalysts to promote the Hantzsch and Biginelli coupling towards DHPMs and DHPs. ${ }^{32}$ The reaction of benzaldehyde, methyl acetoacetate and ammonium acetate using IRMOF-3 as catalyst under solvent-free conditions afforded DHP with 90\% yield (Scheme 5). On the other hand, this catalyst was also tested for the synthesis of DHPMs, observing also high yields of the desired products (Scheme 5). It is however, worth noting that leaching and reusability data, necessary to support the heterogeneity of the process as well as catalyst stability, were not studied.

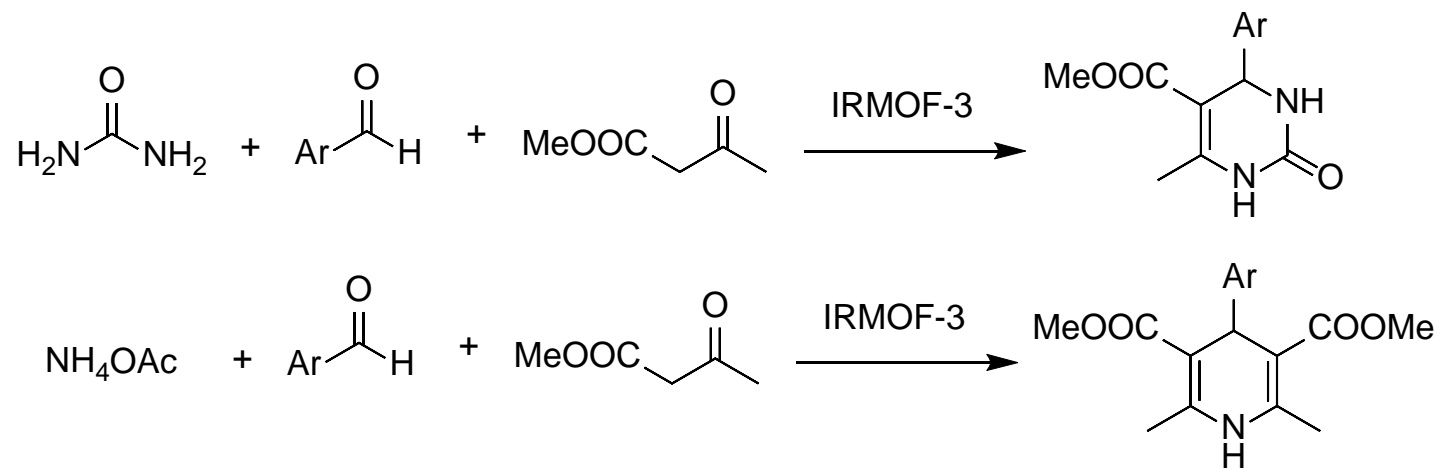

Scheme 5. Synthesis of DHPs and DHPMs using IRMOF-3 as catalyst.

In another precedent, IRMOF-3 was reported as a catalyst for the synthesis of hydroquinoline (Scheme 6) in moderate to high yields under solvent-free conditions. ${ }^{33}$ The catalyst was reused four times without change in the yield for the first three runs, but, however, 
the yield from third to fourth run decreased from 90 to $72 \%$. Unfortunately, there was no data available on the used catalyst that could shed light on the reasons of catalyst deactivation.

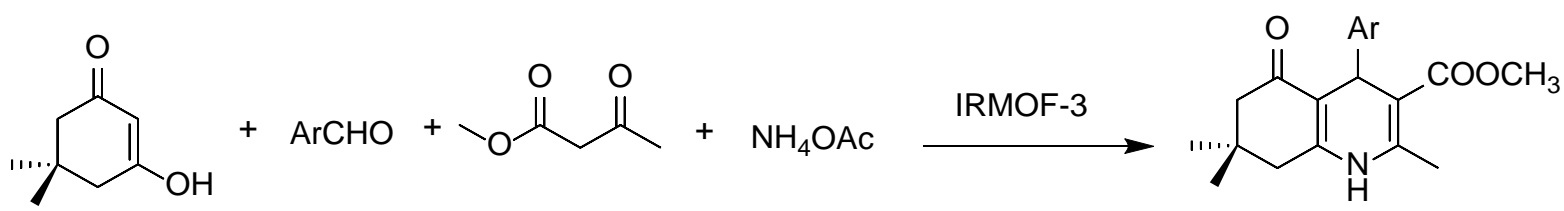

Scheme 6. IRMOF-3 catalyzed synthesis of polyhydroquinoline.

MIL-101(Cr) is a robust MOF whose crystal structure defines two types of cages of 29 and $34 \AA$ diameter that can be accessed through five and six member ring windows. Keggintype phosphotungstic acid $\left(\mathrm{H}_{3} \mathrm{PW}_{12} \mathrm{O}_{40}\right.$, PTA) was encapsulated in the mesocages of MIL-101 (PTA@MIL-101) and its catalytic activity was studied in three-component Biginelli condensation between aldehydes, 1,3-dicarbonyl compounds and urea or thiourea under solvent-free conditions. ${ }^{34}$ Interestingly, PTA@MIL-101 was extremely active to promote the reaction of substituted benzaldehydes with methyl acetoacetate and thiourea to provide various DHPM derivatives. However, hot filtration test revealed the presence of PTA in the liquid phase, thus contributing to the yield increase after the removal of catalyst. Nevertheless, the reusability data indicated that there was no decay in efficiency of the catalyst up to three runs.

\subsection{Friedel-Crafts Alkylation}

A series of anionic MOFs in which the lattice has an excess of negative charge that has to be compensated by the presence of a charge balancing cation in the internal pores was reported as efficient heterogeneous catalysts for the Michael addition of pyrrole to $\beta$-nitrostyrenes (Scheme 7) under solvent-free conditions to afford the corresponding adducts in good to excellent yields. ${ }^{35}$ Initially, the catalytic activity of an anionic MOF, $[\mathrm{HDMA}]_{2}\left[\mathrm{Zn}_{2}(\mathrm{BDC})_{3}(\mathrm{DMA})_{2}\right] .6 \mathrm{DMF} \quad\left(\mathrm{HDMA}^{+}:\right.$dimethylammonium and DMF: N,Ndimethylformamide) was evaluated. The structure of [HDMA $]_{2}\left[\mathrm{Zn}_{2}(\mathrm{BDC})_{3}(\mathrm{DMA})_{2}\right] .6 \mathrm{DMF}$ is defined by $1 \mathrm{D}$ channels along the crystallographic $\mathrm{b}$ axis. These channels with rectangular 
shapes (width and length of about 7.7 and $12.5 \AA$, respectively) are occupied by guest DMF molecules and $\mathrm{HDMA}^{+}$cations. This MOF was subjected to cation exchange to obtain other anionic MOFs with $[\mathrm{TEA}]_{2}\left[\mathrm{Zn}_{2}(\mathrm{BDC})_{3}(\mathrm{DMA})_{2}\right]$ and $[\mathrm{TPA}]_{2}\left[\mathrm{Zn}_{2}-(\mathrm{BDC})_{3}(\mathrm{DMA})_{2}\right]$ with larger organic cations of tetraethylammonium $\left(\mathrm{TEA}^{+}\right)$and tetrapropylammonium $\left(\mathrm{TPA}^{+}\right)$, respectively. It was expected that the acidic properties of these MOFs will decrease by exchanging HDMA with larger organic cations like $\mathrm{TEA}^{+}$and $\mathrm{TPA}^{+}$lacking ammonium protons, thus, enhancing the basicity of the anionic framework. The catalytic activity decreased in the order of $[\mathrm{TPA}]_{2}\left[\mathrm{Zn}_{2}-(\mathrm{BDC})_{3}(\mathrm{DMA})_{2}\right]>[\mathrm{TEA}]_{2}\left[\mathrm{Zn} \mathrm{n}_{2}(\mathrm{BDC})_{3}(\mathrm{DMA})_{2}\right]>$ $[\mathrm{HDMA}]_{2}\left[\mathrm{Zn}_{2}(\mathrm{BDC})_{3}(\mathrm{DMA})_{2}\right] .6 \mathrm{DMF}$ by giving 100,70 and $54 \%$ yield of the Michael adduct under solvent-free conditions at $70{ }^{\circ} \mathrm{C}$. This work clearly demonstrates how MOFs can be tuned to increase their activity, following strategies that have also been used in zeolites. ${ }^{79}$ Using these anionic Zn MOFs, a series of $\beta$-nitrostyrenes were reacted with pyrrole to achieve the target adduct in high yields (> $90 \%$ ) under solvent-free condition, reaching $100 \%$ yield. It was proposed that the high reaction rate was due to the high concentration and availability of reactants inside the MOF pores. $[\mathrm{TPA}]_{2}\left[\mathrm{Zn}_{2}(\mathrm{BDC})_{3}(\mathrm{DMA})_{2}\right]$ was reused for six runs without any loss in its activity.

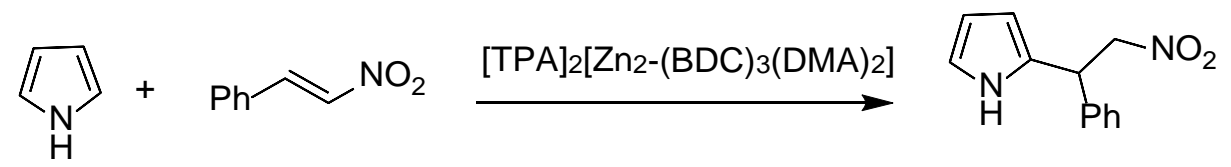

Scheme 7. Michael addition between pyrrole and $\beta$-nitrostyrene catalyzed by $[\mathrm{TPA}]_{2}\left[\mathrm{Zn}_{2}(\mathrm{BDC})_{3}(\mathrm{DMA})_{2}\right]$

In this context, in another precedent, $\mathrm{Cu}_{3}(\mathrm{BTC})_{2}$ has been reported as heterogeneous catalyst for the Michael addition between $\beta$-nitrostyrene and indole, achieving $98 \%$ yield of the adduct under solvent-free conditions (Scheme 8). ${ }^{36}$ This activity of $\mathrm{Cu}_{3}(\mathrm{BTC})_{2}$ is comparable with that of UiO-67-Squar/bpdc $\mathrm{MOF}^{80}$ and $\mathrm{Cu}(\mathrm{dbda})$ using squaramide ligand ${ }^{81}$ in chloroform for the same reaction time. 


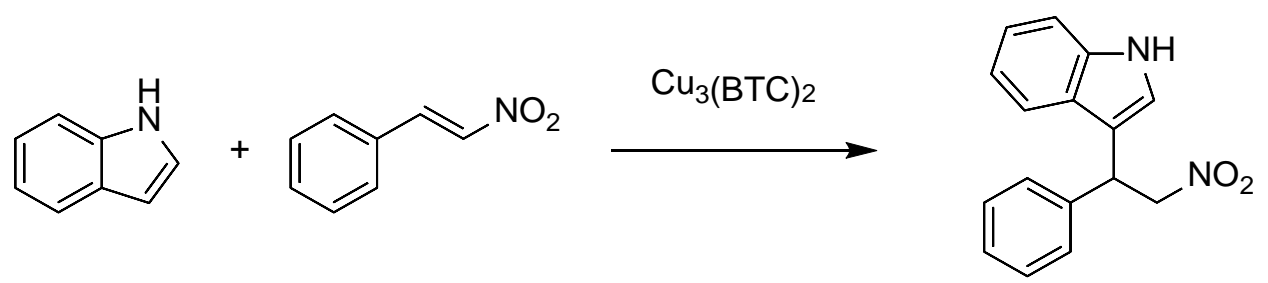

Scheme 8 . Michael addition of indole to $\beta$-nitrostyrene catalyzed by $\mathrm{Cu}_{3}(\mathrm{BTC})_{2}$.

\subsection{Knoevenagel Condensation}

The catalytic activity of anionic MOFs, namely $[\mathrm{TPA}]_{2}\left[\mathrm{Zn}_{2}-(\mathrm{BDC})_{3}(\mathrm{DMA})_{2}\right]$, $[\mathrm{TEA}]_{2}\left[\mathrm{Zn}_{2}(\mathrm{BDC})_{3}(\mathrm{DMA})_{2}\right]$ and $[\mathrm{HDMA}]_{2}\left[\mathrm{Zn}_{2}(\mathrm{BDC})_{3}(\mathrm{DMA})_{2}\right] .6 \mathrm{DMF}$ was also investigated in the Knoevenagel condensation reaction between benzaldehyde and malononitrile (Scheme 9), observing $100 \%$ yield of the condensation product in 10, 35 and $60 \mathrm{~min}$, respectively, under solvent-free conditions at room temperature. ${ }^{37}$ It is interesting to note that low to moderate yields were observed for this reaction in the presence of solvents like acetonitrile, toluene and dichloromethane, but, however, $100 \%$ yield is achieved under solvent-free conditions. In addition, $[\mathrm{TPA}]_{2}\left[\mathrm{Zn}_{2}(\mathrm{BDC})_{3}-(\mathrm{DMA})_{2}\right]$ could be reused in nine runs without any loss in its activity. On the other hand, the reaction of benzaldehyde with ethyl cyanoacetate led to the formation of the desired product in $3 \%$ yield. These activity data can be easily rationalized assuming the occurrence of the reaction predominantly within the pores of MOFs.

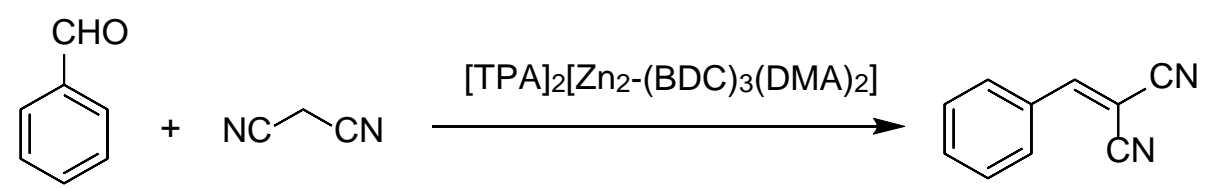

Scheme 9. Knoevenagel condensation reaction between benzaldehyde and malononitrile using $[\mathrm{TPA}]_{2}\left[\mathrm{Zn}_{2}-(\mathrm{BDC})_{3}(\mathrm{DMA})_{2}\right]$ as catalyst.

\subsection{Huisgen 1,3-Dipolar Cycloaddition}

Huisgen 1,3-dipolar cycloaddition of organic azides to terminal alkynes is one of the most convenient synthetic routes to obtain triazole derivatives. The conventional Huisgen 
uncatalyzed thermal methods result in the formation of a $1: 1$ mixture of $1,4-$ and $1,5-$ regioisomers. ${ }^{82}$ A highly regioselective Huisgen 1,3-dipolar cycloaddition between azides and alkynes in the presence of $\mathrm{Cu}(\mathrm{I})$ species has been reported independently by Sharpless ${ }^{83}$ and Meldal $^{84}$ under mild conditions to afford 1,4-disubstituted 1,2,3-triazoles. Later, this reaction has been recognized as $\mathrm{Cu}(\mathrm{I})$-mediated click reaction which has found myriad of applications in modern synthesis. ${ }^{85-89}$ Besides the interest of 1,2,3-triazoles by themselves due to their wide range of biological activity finding various applications as pharmaceuticals and agrochemicals, a large part of the interest of these pentacyclic heterocycles derives from organic synthesis and the possibility to join two independent units to form under mild conditions smart functional molecules and materials. ${ }^{90-92}$ In this aspect, a new Cu-MOF was reported to act as heterogeneous catalyst for the azide-alkyne cycloaddition under solvent-free conditions. ${ }^{38}$ The Cu-MOF was synthesized from copper acetate and ptz and nsa under hydrothermal conditions. The molecular formula of the resulting $\mathrm{Cu}$ MOF was $\mathrm{Cu}(\mathrm{ptz})(\mathrm{nsa})_{0.5} \cdot \mathrm{H}_{2} \mathrm{O}$. The oxidation state of $\mathrm{Cu}$ in this MOF was ascertained to be +1 as evidenced by XPS. Under the optimized reaction conditions at room temperature $(8 \mathrm{~h})$ or at $50{ }^{\circ} \mathrm{C}(2.5 \mathrm{~h})$, the reaction of phenylacetylene and benzyl azide resulted in 92 and $94 \%$ yields of the triazole under solvent-free conditions (Scheme 10). The catalyst was reused for five cycles claiming no significant loss in activity, although the $4^{\text {th }}$ and $5^{\text {th }}$ cycles required slightly longer time to reach complete conversion than those in the previous cycles. This longer time to complete reaction is, however, a clear sign of catalyst deactivation although the origin was not addressed.

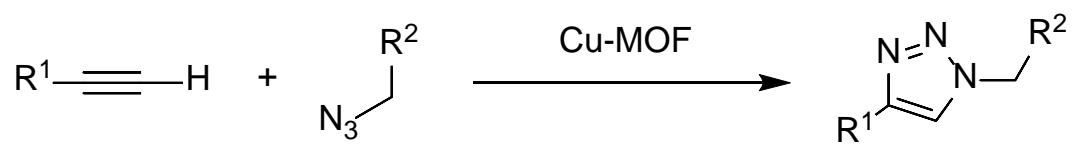

Scheme 10. Synthesis of substituted 1,2,3-triazoles using Cu-MOF as solid catalyst.

\subsection{Propargylamine Synthesis}


Propargylamine is considered an important motif that can be found in many natural products and therapeutic drugs. ${ }^{93}$ In addition, they are also used as one of the key intermediates for the synthesis of many nitrogen-containing natural products and biologically active compounds, such as $\beta$-lactams ${ }^{94}$ and tremorine analogues. ${ }^{95}$ Due to the interest of this type of nitrogen compounds, a new $\mathrm{Cu}(\mathrm{I})-\mathrm{MOF}$ has been developed for the synthesis of propargylamine via the three-component coupling of secondary amines, alkynes, and aromatic aldehydes under solvent-free conditions (Scheme 11). ${ }^{39}$ Heterogeneity test confirmed the absence of leaching of relevant catalytic species. The catalyst was reused five cycles with no considerable decrease in the final yield.

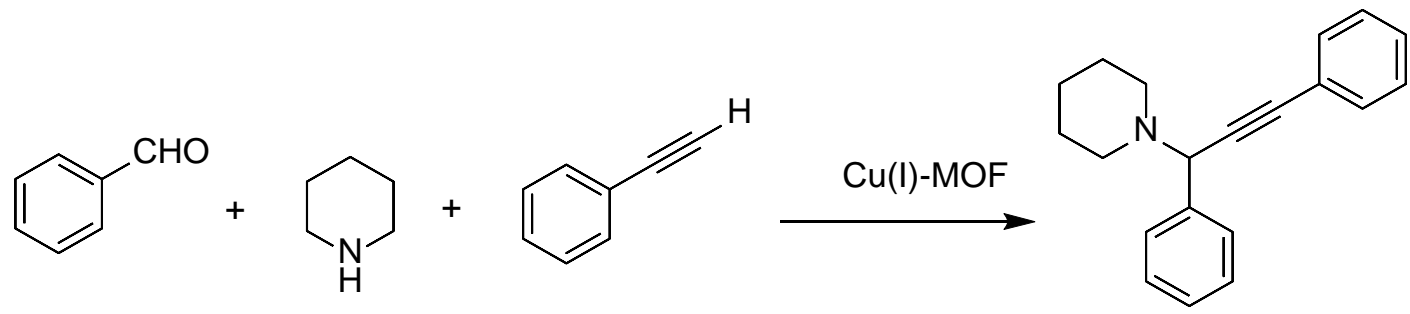

Scheme 11. Cu(I) MOF catalyzed propargylamine.

\section{7 $\mathrm{CO}_{2}$ Cycloaddition to Epoxides}

$\mathrm{CO}_{2}$ can be considered as a cheap, nontoxic, non-flammable and abundant feedstock in the synthesis of some important chemical products. ${ }^{96-99}$ In the context of $\mathrm{CO}_{2}$ fixation, one of the most convenient synthetic intermediate are cyclic carbonates obtained by the cycloaddition of $\mathrm{CO}_{2}$ to epoxides. ${ }^{100,} 101$ Cyclic carbonates can be adequate precursors for producing polycarbonates and other fine chemicals. Although many homogeneous and heterogeneous catalytic systems have been reported for the cycloaddition of $\mathrm{CO}_{2}$ to epoxides, ${ }^{102-107}$ the use of solid catalysts is advantageous from the point of view of simplification of the work-up in the separation process. ${ }^{108}$

Moving towards the next section in which incorporation of ionic liquids (ILs) inside the MOFs pores will be described. Organic tetraalkylammonium halides have been used in 
combination of MOFs to promote $\mathrm{CO}_{2}$ insertion to epoxides. Thus, MIL-101(Cr) has been reported as a heterogeneous catalyst promoting the solvent-free coupling of $\mathrm{CO}_{2}$ with styrene oxide to produce styrene carbonate. ${ }^{40}$ The presence of tetrabutylammonium bromide as cocatalyst was essential for the formation of the styrene carbonate. On the other hand, cycloaddition of $\mathrm{CO}_{2}$ to propylene oxide resulted in propylene carbonate. The catalyst stability was, however, insufficient and the two times reused catalyst loosed its crystallinity as evidenced from powder XRD.

An amino group functionalized IRMOF-3 (F-IRMOF-3; Scheme 12) was reported as heterogeneous catalyst for the solvent-free synthesis of cyclic carbonates without any cocatalyst reaching TOF values of $778 \mathrm{mmolg}^{-1} \mathrm{~h}^{-1} \cdot{ }^{41}$ Furthermore, F-IRMOF-3-4d resulted in better catalytic activity than other reported catalysts like MIXMOF/Et ${ }_{4} \mathrm{NBr}\left(499 \mathrm{mmolg}^{-1} \mathrm{~h}^{-}\right.$ $\left.{ }^{1}\right),{ }^{109} \mathrm{MgO} \quad\left(0.9 \mathrm{mmolg}^{-1} \mathrm{~h}^{-1}\right),{ }^{110} \mathrm{SmOCl}\left(4.1 \mathrm{mmolg}^{-1} \mathrm{~h}^{-1}\right),{ }^{111}$ cross-linked polymeric nanoparticles (127 mmolg $\left.^{-1} \mathrm{~h}^{-1}\right){ }^{112}$ hydroxyl IL grafted onto cross-linked divinylbenzene polymer $\left(70 \mathrm{mmolg}^{-1} \mathrm{~h}^{-1}\right)^{113}$ and polystyrene-supported threonine $\left(16 \mathrm{mmolg}^{-1} \mathrm{~h}^{-1}\right) .{ }^{114}$ F-IRMOF-3-4d was reused for four runs without much change in its catalytic activity.

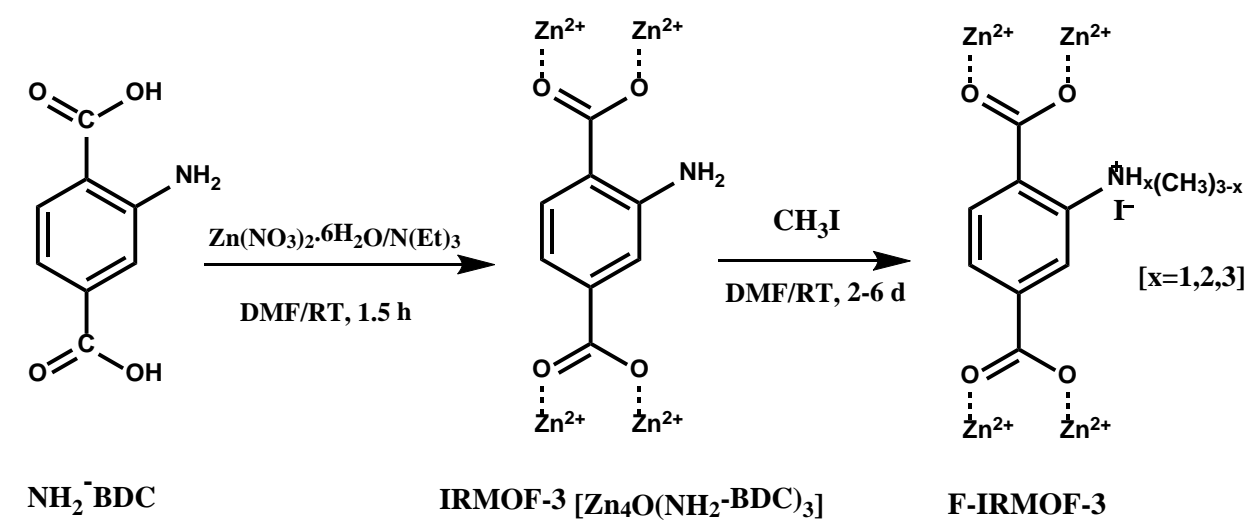

Scheme 12. Synthesis of F-IRMOF-3 from $\mathrm{NH}_{2}$-BDC.

A 3D MOF, [ $\left.\mathrm{Zn}_{4} \mathrm{O}(2,6-\mathrm{NDC})(\mathrm{BTB})_{4 / 3}\right]\left(\mathrm{MOF}-205, \mathrm{BET}=4200 \mathrm{~m}^{2} / \mathrm{g}\right)$ was synthesised by microwave heating and its catalytic activity was examined in the cycloaddition of $\mathrm{CO}_{2}$ to 
propylene oxide to produce the corresponding five-membered cyclic carbonates under solventfree conditions. ${ }^{42}$

All the above data show that frequently MOFs can be used as solid catalysts in the absence of solvents for condensations, Michael addition, cycloadditions and couplings giving yields that are even better than in the presence of solvents. Although it should be commented that catalysis in the absence of solvent is not specific of MOFs and most of the above discussed reactions have also been conducted with other solid catalysts, the present review aims to illustrate that under solvent-free conditions MOFs can exhibit even higher catalytic activity than in the presence of solvents. An additional advantage is the environmental benefits derived from the diminution of wastes associated to the use of solvents.

\section{IL@MOFs}

Due to their low vapor pressure, ILs are considered as green solvents for various applications including reaction media, ${ }^{115,116}$ extractions, ${ }^{117}$ catalysis, ${ }^{118,}{ }^{119}$ gas absorption ${ }^{120}$ and electrolytes in electrochemical devices. ${ }^{121}$ Although the use of ILs as alternative media to conventional organic solvents is well documented, ${ }^{122}$ an appealing approach is to impregnate ILs onto porous materials like MOFs forming a thin, micrometric film coating the walls of the solid. Due to Coulombic and polar interactions, the IL film remains on the surface of the material, modifying the environment of the surface active sites, even if a bulk liquid solvent, immiscible with the IL, is present in the system. In this context, a recent review has summarized the different strategies employed for the incorporation of ILs over MOFs and their applications including gas absorption. ${ }^{23}$ Hence, the present review restricts itself to the catalytic applications of ILs supported on MOFs. The following section will describe those reactions catalysed by MOF embedding ILs, frequently having acid or basic groups to cooperate in the reaction mechanism. 


\subsection{Knoevenagel Condensation}

Task-specific ionic liquids (TSILs) have been reported as environmentally benign solvents and highly active co-catalysts in Knoevenagel condensation. ${ }^{123,124}$ However, the use of TSILs in homogeneous catalysis or liquid-liquid biphasic system is hampered by the difficulty in the separation and recycling of the catalyst. Another problem is that the reaction mixture becomes also inevitably contaminated with byproducts formed from the consecutive reaction of the primary product in contact with the homogeneous catalyst. In this regard, these limitations together with an economy in the amount of TSIL needed can be achieved by coating a porous surface with a thin film of the IL.

As an example to illustrate this approach, a size- and shape-selective porous catalyst embedding IL, namely HKUST-1 incorporating an amino-functionalized basic ionic liquid (ABIL-OH/HKUST-1) was synthesized by impregnation (Scheme 13). ${ }^{43}$ Characterization of this catalyst revealed that ABIL-OH is likely confined within the nanocavities of HKUST-1 via a $\mathrm{Cu}-\mathrm{NH}_{2}$ coordination bond. The fact that the ABIL-OH/HKUST-1 catalyst exhibits visually a pale blue colour was taken as evidence of the change in the coordination of copper ions as the colour is associated with the $\mathrm{d}$-d transition of $\mathrm{Cu}^{2+}$ ions. Furthermore, XPS studies of ABIL-OH/HKUST-1 indicate that the $\mathrm{Cu} 2 \mathrm{p}_{3 / 2}$ and $\mathrm{Cu} 2 \mathrm{p}_{1 / 2}$ binding energy shifted from $933.8 \mathrm{eV}$ to $933.3 \mathrm{eV}$ and $953.5 \mathrm{eV}$ to $953.0 \mathrm{eV}$, respectively. This slight reduction of binding energy is in agreement with the occurrence of an electron transfer from $\mathrm{NH}_{2}$ to $\mathrm{Cu}^{2+}$ centre. The loading of ABIL-OH in ABIL-OH/HKUST-1was determined to be $1.07 \mathrm{mmol} \mathrm{g}^{-1}$ by elemental analysis.

The catalytic activity of ABIL-OH/HKUST-1 was evaluated in the Knoevenagel condensation between benzaldehyde and malononitrile. The as-synthesized HKUST-1 resulted in $55.3 \%$ benzaldehyde conversion after $240 \mathrm{~min}$. On the other hand, ABIL-OH/HKUST-1 
catalyst exhibited complete benzaldehyde conversion after 210 min under the same conditions. Meanwhile, the use of ABIL-OH as homogeneous organocatalyst showed complete benzaldehyde conversion after 120 min. This high activity of ABIL-OH could be due to the availability of free $\mathrm{NH}_{2}$ group in ABIL-OH, thus, acting as Lewis base catalyst. ABILOH/HKUST-1 showed high catalytic activity (> $90 \%$ conversion) with $100 \%$ selectivity after five recycles, but, however, a slight decay in conversion is observed, indicating again the occurrence of some deactivation. On the other hand, comparison of the powder XRD between the fresh and reused catalyst revealed that the main diffraction peaks are still observed in reused catalyst, although the disappearance of several peaks at high angle was observed indicating some changes in the MOF structure. It has been observed frequently that HKUST-1 has an instable structure that can collapse due to the strong binding of $\mathrm{Cu}^{2+}$ with substrates, reagents or solvents. ${ }^{125,126}$ However, hot filtration test indicated the heterogeneity of the process without clues of $\mathrm{Cu}$ leaching. No catalytic activity was observed for the reaction between o-hydroxybenzaldehyde $(6.65 \times 5.41 \AA$ ) and 3-methoxy-4-hydroxybenzaldehyde $(7.60 \times 6.43$ $\AA$ ) with malononitrile in the presence of ABIL-OH/HKUST-1, whereas these substrates were converted completely to their respective products with ABIL-OH as homogeneous catalyst under identical reaction conditions. This activity difference was attributed to the confinement of the active site within the microenvironment of HKUST-1.
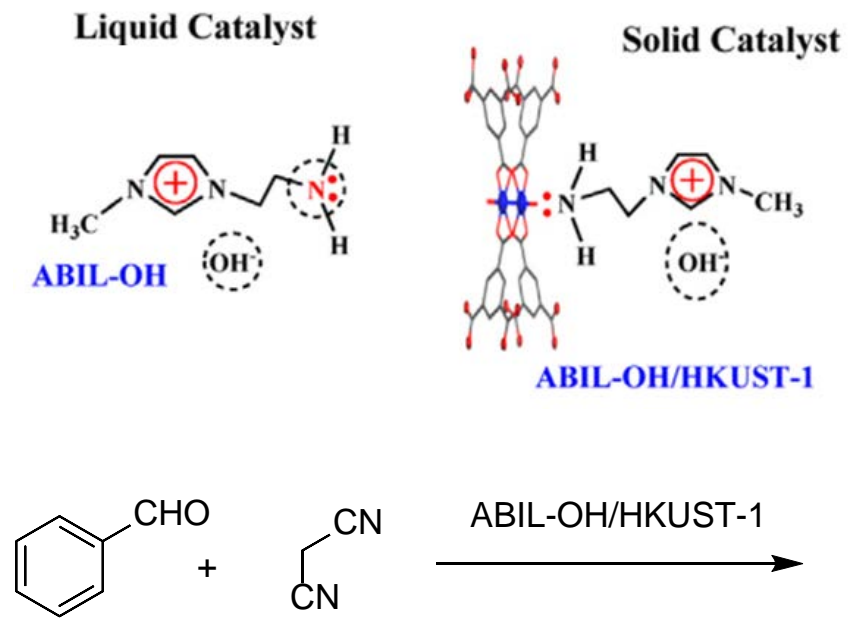<smiles>N#CC(C#N)=Cc1ccccc1</smiles> 
Scheme 13. Structure and proposed interaction of ABIL-OH with HKUST-1 and Knoevenagel condensation catalyzed by ABIL-OH/HKUST-1. Reproduced from ref ${ }^{43}$ with the permission from Elsevier.

\subsection{Oxidation Reactions}

ILs have been among the preferred green solvents to carry out catalytic reactions promoted by transition metals and metal nanoparticles. ${ }^{127}$ In this regard, $\mathrm{Cu}_{3}(\mathrm{BTC})_{2}$ has been employed as a support to immobilize $\mathrm{PdCl}_{2}$ with the aid of ionic liquids such as 1-carboxyethyl3-methylimidazolium chloride $\left(\mathrm{PdCl}_{2}-\mathrm{ILs} / \mathrm{Cu}_{3}(\mathrm{BTC})_{2}\right) .{ }^{44}$ The catalytic activity of the as-synthesized $\mathrm{PdCl}_{2}$-ILs/Cu $3(\mathrm{BTC})_{2}$ catalyst was studied in the selective oxidation of cyclohexene by molecular oxygen as oxidant and t-butylhydroperoxide (TBHP) as initiator. The use of $\mathrm{PdCl}_{2}-\mathrm{ILs} / \mathrm{Cu}_{3}(\mathrm{BTC})_{2}$ catalyst afforded $24 \%$ conversion of cyclohexene with a maximum selectivity of $53.2 \%$ to 2-cyclohexene-1-one. The other products formed in this reaction are 2-cyclohexene-1-ol and t-butyl-2-cyclohexenyl-1-peroxide. The corresponding epoxide was not detected. Further, it was observed that the selectivity towards 2-cyclohexene1-one achieved with $\mathrm{PdCl}_{2}$-ILs/CNTs (14\%) and $\mathrm{PdCl}_{2}$-ILs/SBA-15 (10 \%) is lower than with $\mathrm{PdCl}_{2}-\mathrm{ILs} / \mathrm{Cu}_{3}(\mathrm{BTC})_{2}$ catalyst. This enhanced activity of the later catalyst is believed to be due to the Pd-Cu cooperative effect. Electron paramagnetic resonance (ESR) suggested that the most probable radical species should be TBHP-derived oxygen centred radical according to earlier precedent. ${ }^{128}$ This proposal of the involvement of oxygen radical initiator was further verified by a strong inhibition in cyclohexene conversion when the oxidation of cyclohexene is performed with 2,6-di-t-butyl-methylphenol as radical scavenger. ${ }^{129}$ Inductively coupled plasma (ICP) analysis showed the existence of copper and palladium in the solution as 127 and 223 ppm, respectively, indicating that the metal leaching occurs in a very limited extent. The catalyst was recycled three times without appreciable change in conversion and selectivity of 
2-cyclohexene-1-one. Powder XRD showed the collapse of the crystalline structure of the $\mathrm{Cu}$ MOF. The catalytic activity of $\mathrm{PdCl}_{2}-\mathrm{ILs} / \mathrm{Cu}_{3}(\mathrm{BTC})_{2}$ was superior than $\mathrm{PdCl}_{2} / \mathrm{Cu}_{3}(\mathrm{BTC})_{2}$ in terms of the reaction rate and TOF value. The reaction rate and TOF value for the later catalyst was $0.4 \mathrm{~mol} \mathrm{~g}^{-1} \mathrm{~h}^{-1}$ and $6.7 \mathrm{~h}^{-1}$, respectively, while the reaction rate and TOF value achieved for the former catalyst is $0.54 \mathrm{~mol} \mathrm{~g}^{-1} \mathrm{~h}^{-1}$ and $8.1 \mathrm{~h}^{-1}$, respectively. These activity data indicate the benefits of using ILs, probably because they favour the uniform dispersion of $\mathrm{PdCl}_{2}$ species on the interior of the MOF. On the other hand, a significant decrease in conversion was noticed when $\mathrm{PdCl}_{2} / \mathrm{Cu}_{3}(\mathrm{BTC})_{2}$ was used as catalyst during reusability experiments.

Recently, UiO-66 was loaded with different percentages of 1-methylimidazolium-3propylsulfonate hydrogensulfate and its activity was studied in the oxidative desulfurization using hydrogen peroxide as oxidant. ${ }^{45}$ It was observed that at $40{ }^{\circ} \mathrm{C}$ the sulfur removal is higher than $94 \%$ with an O/S molar ratio of 7:1 using $40 \%$ 1-methylimidazolium-3-propylsulfonate hydrogensulfate supported on UiO-66. Increasing the content of 1-methylimidazolium-3propylsulfonate hydrogensulfate lowered the activity to $92.9 \%$ under identical conditions. Reusability experiments showed that the sulphur removal was $90.6 \%$ after the sixth cycle, thus, showing the stability of the catalytic activity under the experimental conditions used. However, no data were given on leaching or the structural integrity of the reused sample compared to the fresh catalyst.

Recently, phosphotungstic acid $\left(\mathrm{H}_{3} \mathrm{PW}_{12} \mathrm{O}_{40}, \mathrm{PW}\right)$ was immobilized within the nanocages of DAIL-modified MIL-101(Cr) to obtain PW/DAIL/MIL-101(Cr) and its activity was examined in the oxidation of benzyl alcohol using TBHP. ${ }^{46}$ An analogous catalyst, namely, PW/MIL101(Cr) was also prepared as a control and its activity was compared with that PW/DAIL/MIL101(Cr) to gain information on the role of DAIL during this oxidation reaction. Activated MIL101(Cr) was treated with DAIL to obtain DAIL/MIL-101(Cr) in which a strong coordination bond between the metal centres of the MOF and the amino groups of the DAIL was proposed 
to occur. The immobilization of PW onto DAIL/MIL-101(Cr) was performed through anion exchange of the corresponding poly oxometal and the DAIL. The PW cluster has an appropriate dimension to diffuse through the larger windows of MIL-101(Cr) of $12 \AA$. The preparation procedure followed to obtain PW/DAIL/MIL-101(Cr) and the chemical structure of DAIL are illustrated in Figure 1. The powder XRD pattern of DAIL/MIL-101(Cr) was in good agreement with that of MIL-101(Cr), confirming that the crystal structure remains mostly intact after the functionalization of the MOF with DAIL cations. It was observed that the thermal stability of PW/DAIL/MIL-101(Cr) increased compared to that of DAIL/MIL-101(Cr) and this enhanced stability was proposed to be due to the additional interactions between DAIL and PW molecules. The XRF measurements indicated that the loading values of PW were $0.18 \mathrm{mmol}$ $\mathrm{g}^{-1}$ and $0.23 \mathrm{mmol} \mathrm{g}^{-1}$ for the HPW/MIL-101(Cr) and PW/DAIL/MIL-101(Cr), respectively. PW/DAIL/MIL-101(Cr) exhibited higher catalytic activity (95 \% conversion and 1900 TON) than HPW/MIL-101(Cr) (70 \% conversion and 1400 TON) in the oxidation of benzyl alcohol to benzaldehyde. On the other hand, the conversion of benzyl alcohol was influenced by the loading of DAIL, observing that benzyl alcohol conversion gradually increased when DAIL loading increases from 0.3 to $0.9 \mathrm{mmol} \mathrm{g}^{-1}$. These data suggest that the presence of DAIL group is essential to achieve high activity. In contrast, MIL-101(Cr) gave $43 \%$ benzyl alcohol conversion under identical conditions. Hot filtration test proved the heterogeneity of the process. The catalyst was reused five times with no significant decrease in the catalytic activity. The similar powder XRD patterns of the fresh material and reused PW/DAIL/MIL-101(Cr) catalyst indicates that the system is stable under the reaction conditions. Although high conversion of benzyl alcohol was observed with this catalytic system in chloroform, it is clear that the use of volatile organic solvents, particularly chlorinated solvents do not comply the principles of green chemistry and further work is necessary to replace these liquids by more environmentally tolerable solvents. In another precedent, the catalytic performance of 
$\mathrm{PW}_{4} / \mathrm{DAIL} / \mathrm{MIL}-100(\mathrm{Fe})$ was compared with that of the DAIL free catalyst (HPW $/ \mathrm{MIL}$ $100(\mathrm{Fe})$ in the oxidation of benzyl alcohol using TBHP as an oxidant. ${ }^{47}$ It was interesting to note that the TON value of former catalyst is 613 , which is superior to the later catalyst with the value of 433 under identical conditions and this higher activity is ascribed to the ability of DAIL groups to form hydrogen bonds, enhancing the accessibility of TBHP.
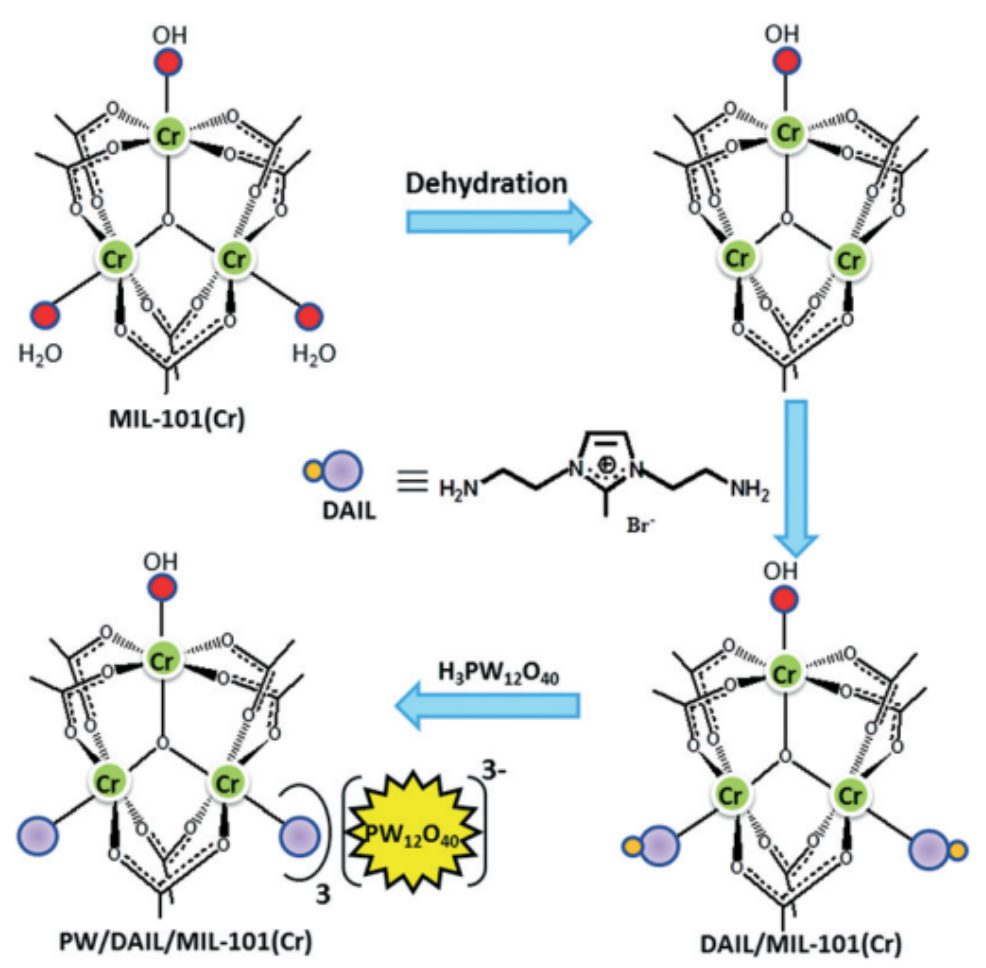

Figure 1. Structure of DAIL and synthesis of PW/DAIL/MIL-101(Cr) catalyst. Reproduced from ref ${ }^{46}$ with the permission from Royal Society of Chemistry.

\subsection{Esterification Reactions}

A magnetically recoverable and efficient catalyst was prepared for biodiesel synthesis (esterification of oleic acid with ethanol) by confining the Brønsted ionic liquid 1,4-butanediyl3,3'-bis(3-sulfopropyl)imidazolium bis(hydrogensulfate) through the amino groups within an amino-functionalized magnetic MOF composite $\left(\mathrm{Fe}_{3} \mathrm{O}_{4} @ \mathrm{NH}_{2}-\mathrm{MIL}-88 \mathrm{~B}(\mathrm{Fe})\right)$ as shown in Figure $2 .{ }^{48}$ The use of $\mathrm{Fe}_{3} \mathrm{O}_{4} @ \mathrm{NH}_{2}-\mathrm{MIL}-88 \mathrm{~B}(\mathrm{Fe})$ as catalyst for the esterification of oleic acid 
with ethanol resulted in $8.1 \%$ yield, which was believed to be due to the presence of unsaturated $\mathrm{Fe}^{\mathrm{III}}$ metal centres acting as Lewis acid sites. ${ }^{130}$ On the other hand, the use of homogeneous counterpart, namely, ionic liquid DAIL showed $90.3 \%$ yield that is similar, but, lower, than that of the magnetic heterogeneous catalyst DAIL-Fe $\mathrm{O}_{4} @ \mathrm{NH}_{2}-\mathrm{MIL}-88 \mathrm{~B}(\mathrm{Fe})$ (acidity of $1.76 \mathrm{mmol} \mathrm{H}^{+} \mathrm{g}^{-1}$; DAIL loading amount of $0.58 \mathrm{mmol} / \mathrm{g}$ ) that afforded $93.2 \%$ yield. Interestingly, the catalytic activity of DAIL-Fe $\mathrm{O}_{4} @ \mathrm{NH}_{2}-\mathrm{MIL}-88 \mathrm{~B}(\mathrm{Fe})$ was comparable with earlier reported acidic catalysts (80-93 \% yields). ${ }^{131-136}$ The advantage of the present magnetic MOF catalyst is not only that DAIL- $\mathrm{Fe}_{3} \mathrm{O}_{4} @ \mathrm{NH}_{2}-\mathrm{MIL}-88 \mathrm{~B}(\mathrm{Fe})$ showed slightly higher catalytic activity, but its convenient and efficient separation by applying a weak magnetic field. However, the catalytic activity of DAIL-Fe $\mathrm{O}_{4} @ \mathrm{NH}_{2}-\mathrm{MIL}-88 \mathrm{~B}(\mathrm{Fe})$ is still far from perfect, since reusability tests showed that it decreased after six consecutive cycles, something that is not surprising considering the tendency of larger molecules to poison porous materials. The catalysis was nevertheless, found to be heterogeneous in nature.

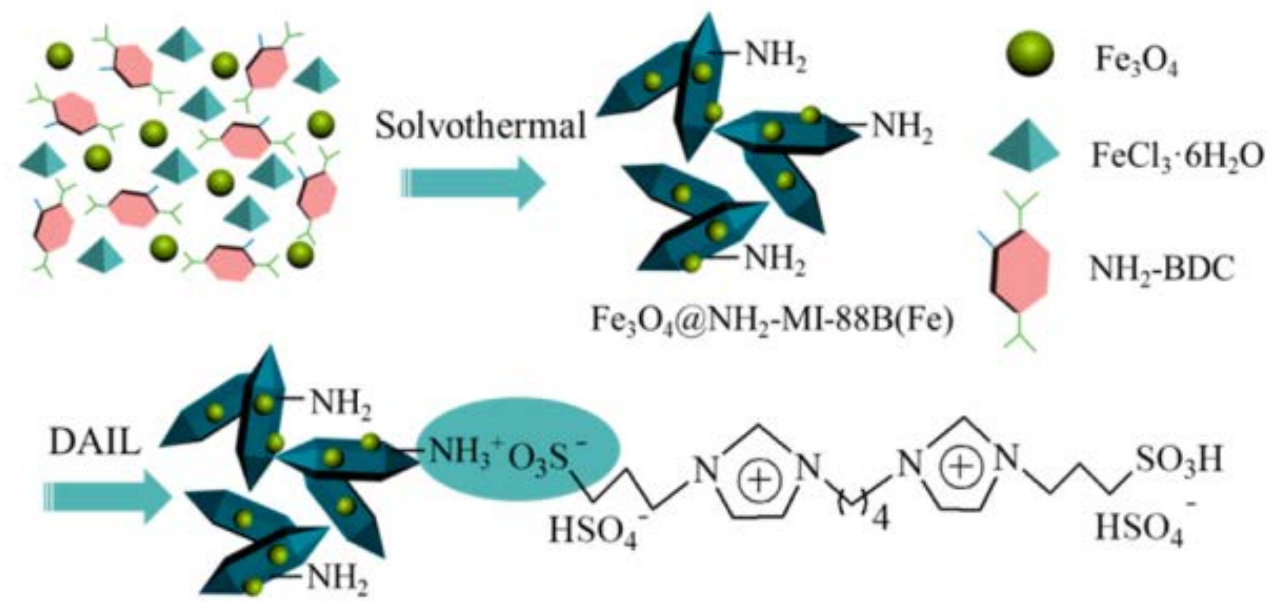

Figure 2. Preparation of functionalized magnetic MOF incorporating acid ionic liquid DAIL$\mathrm{Fe}_{3} \mathrm{O}_{4} @ \mathrm{NH}_{2}-\mathrm{MIL}-88 \mathrm{~B}(\mathrm{Fe})$. Reproduced from ref ${ }^{48}$ with the permission from American Chemical Society.

A new strategy designed to construct the IL, POM, and MOF composite is shown in Figure 3. In this approach, POM was loaded first within the cages of MIL-100 to play the role 
of a bridge. Then, the IL was introduced to encapsulate the heteropolyanion. ${ }^{49}$ The POM-ILfunctionalized MOF has several advantages such as a simple preparation process, high stability, high content of active components, easy separation and high reusability. Furthermore, the preparation method did not use toxic or harmful reagents such as HF and DMF, thus exhibiting advantages from the environmental point of view. The catalytic activity of $\left[\mathrm{SO}_{3} \mathrm{H}-\left(\mathrm{CH}_{2}\right)_{3}-\right.$ $\mathrm{HIM}]_{3} \mathrm{PW}_{12} \mathrm{O}_{40} @ \mathrm{MIL}-100(\mathrm{Fe})$ was studied as catalyst in the esterification of oleic acid with ethanol. The pristine MIL-100(Fe) showed some catalytic activity of $15.5 \%$ conversion at $111^{\circ} \mathrm{C}$, probably due to the role of coordinatively unsaturated $\mathrm{Fe}^{3+}$ ions as Lewis acid sites. On the other hand, encapsulated phosphotungstate $\mathrm{HPW}_{12} \mathrm{O}_{40} @ \mathrm{MIL}-100$ (Fe) (acidity: $0.39 \mathrm{mmol} \mathrm{H}^{+} \mathrm{g}^{-1}$ ) gave $40.3 \%$ conversion of oleic acid. Importantly, $94.6 \%$ conversion was achieved using $\left[\mathrm{SO}_{3} \mathrm{H}-\left(\mathrm{CH}_{2}\right)_{3}-\mathrm{HIM}\right]_{3} \mathrm{PW}_{12} \mathrm{O}_{40} @ \mathrm{MIL}-100$ as catalyst (acidity: 1.74 mmol H${ }^{+} \mathbf{g}^{-}$ ${ }^{1}$ ). This enhanced activity of the composite catalyst can be attributed to the sulfonic acid groups in the IL cation increasing the number of Brönsted acid sites compared to heteropolyanion. Also, the existence of a cooperative effect between Lewis and Brönsted acid sites improving the activity of the catalyst was proposed. ${ }^{137}$ Although the catalytic activity of the pure IL was higher (95.1\%), the heterogeneous catalyst could be easily separated from the reaction medium. In contrast, $\left[\mathrm{SO}_{3} \mathrm{H}-\left(\mathrm{CH}_{2}\right)_{3}-\mathrm{HIM}\right]\left[\mathrm{HSO}_{4}\right] @ \mathrm{MIL}-100(\mathrm{Fe})$ catalyst lacking POM that was prepared by direct impregnation of MIL-100(Fe) with IL exhibited $90.2 \%$ conversion under identical condition, but, however, its activity decreased upon reuse (Figure 4). Overall, the $\left[\mathrm{SO}_{3} \mathrm{H}-\left(\mathrm{CH}_{2}\right)_{3}-\mathrm{HIM}\right]_{3} \mathrm{PW}_{12} \mathrm{O}_{40} @ \mathrm{MIL}-100(\mathrm{Fe})$ catalyst showed optimal activity for the esterification of oleic acid compared with other catalysts. ${ }^{132,138-141}$ The catalyst was reused for six cycles without appreciable decay in its activity. 

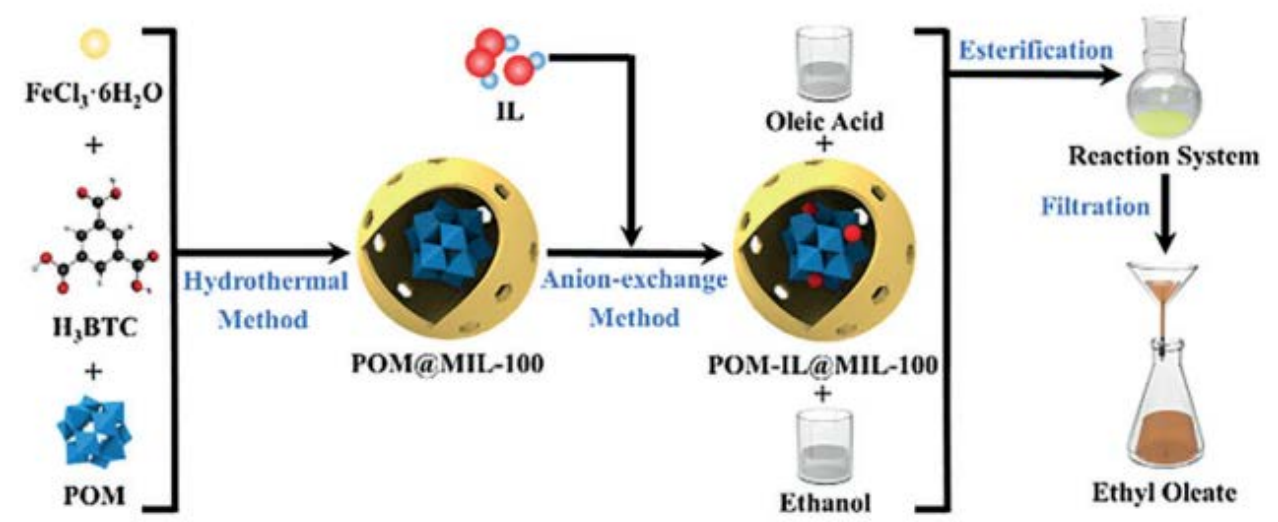

Figure 3. Synthesis of $\left[\mathrm{SO}_{3} \mathrm{H}-\left(\mathrm{CH}_{2}\right)_{3}-\mathrm{HIM}\right]_{3} \mathrm{PW}_{12} \mathrm{O}_{40} @ \mathrm{MIL}-100(\mathrm{Fe})$ and its use as esterification catalyst for the preparation of biodiesel. Reproduced from ref ${ }^{49}$ with the permission from Wiley.

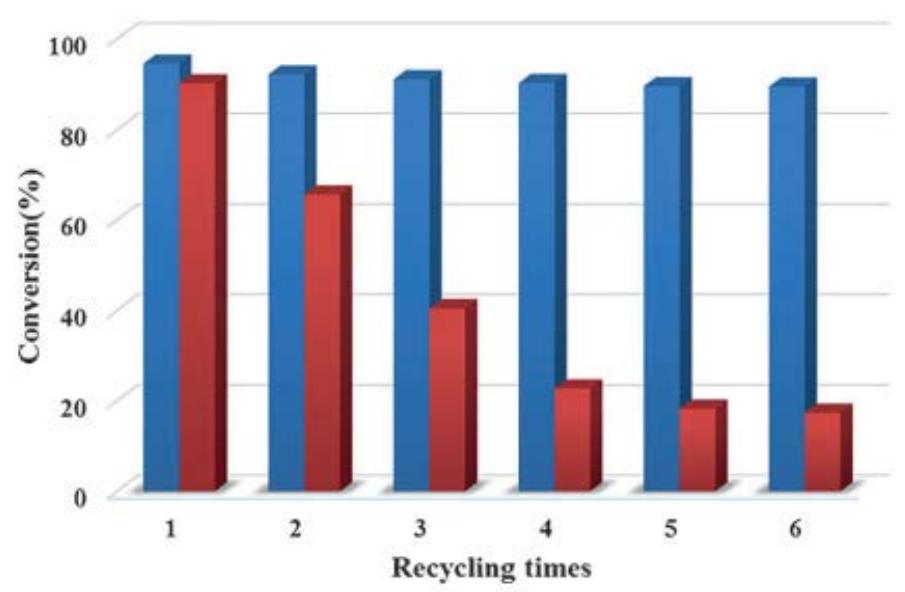

Figure 4. Activity of $\left[\mathrm{SO}_{3} \mathrm{H}-\left(\mathrm{CH}_{2}\right)_{3}-\mathrm{HIM}\right]_{3} \mathrm{PW}_{12} \mathrm{O}_{40} @ \mathrm{MIL}-100$ (blue) and $\left[\mathrm{SO}_{3} \mathrm{H}-\left(\mathrm{CH}_{2}\right)_{3}-\right.$ HIM][HSO4]@MIL-100 (red) upon recycling for the esterification of oleic acid with ethanol. Reproduced from ref ${ }^{49}$ with the permission from Wiley.

A Brönsted IL acid, namely, N-methyl-2-pyrrolidonium methyl sulfonate ([NMP $]^{+} \mathrm{CH}_{3} \mathrm{SO}_{3}{ }^{-}$) was immobilized on MIL-101(Cr) and its catalytic activity was evaluated in the esterification of acetic acid with amyl alcohol. ${ }^{50}$ This catalyst exhibited $82.4 \%$ conversion which is comparable to $[\mathrm{NMP}]^{+} \mathrm{CH}_{3} \mathrm{SO}_{3}{ }^{-}(97 \%)^{142}$ and HPW/MIL-101 (92.3 \%) $)^{143}$ catalysts for the esterification reaction. Although the Brönsted IL acid loaded on MIL-101(Cr) showed slightly lower activity compared to $\mathrm{NMP}]^{+} \mathrm{CH}_{3} \mathrm{SO}_{3}{ }^{-}$and $\mathrm{HPW} / \mathrm{MIL}-101$, the merit of 
MIL-1001(Cr) supported IL is to be a heterogeneous catalysts, avoiding tungstic acid. Furthermore, the catalyst was reused six times without considerable decay in its activity.

Another Bronsted acidic IL (BAIL) confined inside the nanocages of MIL-101(Cr) was reported for the acetalization of benzaldehyde with glycerol (Scheme 14). ${ }^{51}$ The BAIL functionalized MIL-101 catalyst was prepared by heating at reflux temperature imidazole (IMIZ) or triethylene diamine (TEDA) with the dehydrated MIL-101. These precursors were further treated with 1,4-butane sultone and exchanged with $\mathrm{H}_{2} \mathrm{SO}_{4}$ as shown in Figure 5. The parent MIL-101(Cr) showed $32 \%$ conversion of benzaldehyde at $90{ }^{\circ} \mathrm{C}$ after $3 \mathrm{~h}$, this low conversion being probably due to its mild Lewis acidity. Interestingly, the TEDA-BAIL/MIL101 afforded 88\% conversion of benzaldehyde while $58 \%$ of benzaldehyde conversion is achieved with IMIZ-BAIL/MIL-101 catalyst under identical conditions. Furthermore, the catalytic activity achieved by TEDA-BAIL/MIL-101 was comparable with the benchmark catalyst, namely p-toluenesulfonic acid which gives $90 \%$ conversion of benzaldehyde. The catalyst was recycled six times without significant decay in catalytic activity or leaching of active sites.

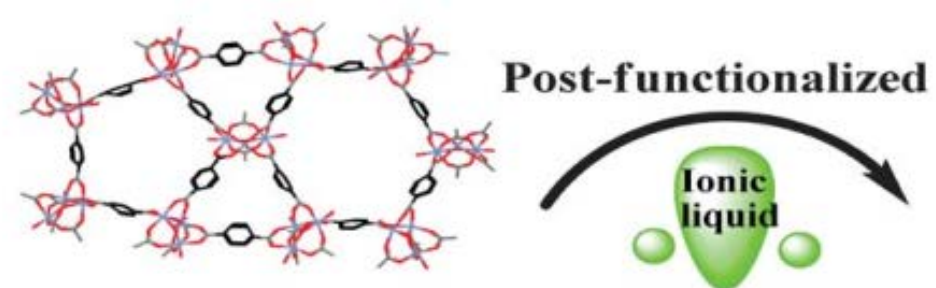

Pure MIL-101

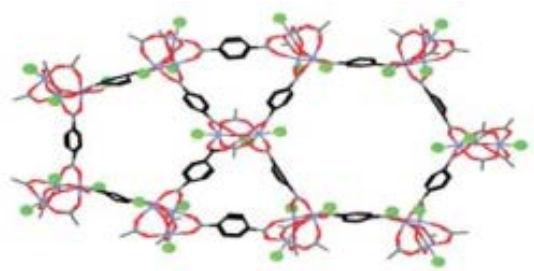

BAIL/MIL-101
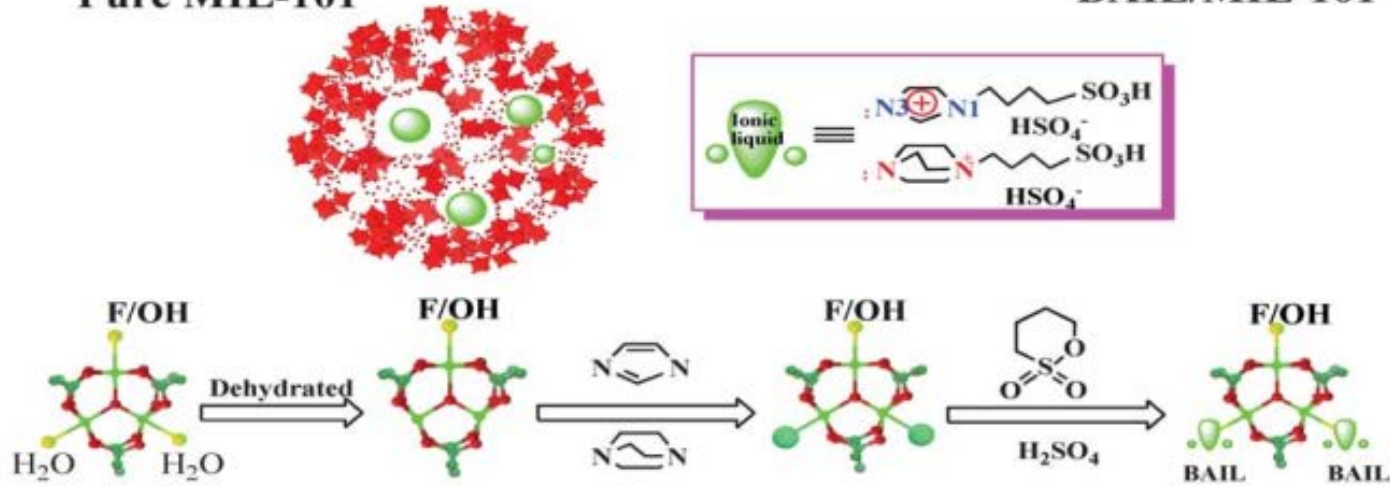
Figure 5. Procedure for the synthesis of BAIL confined in MIL101 nanocages by post-synthetic modification. Reproduced from ref ${ }^{51}$ with the permission from Royal Society of Chemistry.

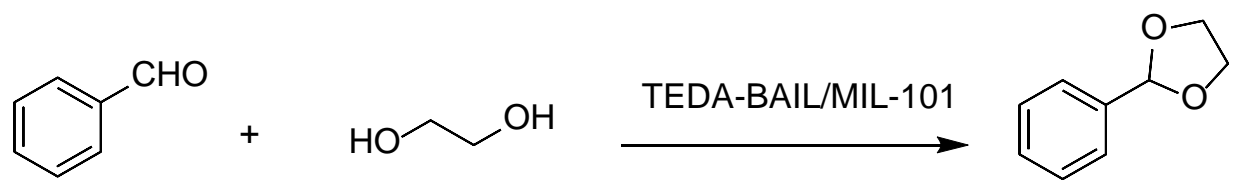

Scheme 14. Acetalization of benzaldehyde with glycerol using TEDA-BAIL/MIL-101 as catalyst.

\subsection{Hydrogenation of Acetylene}

Selective hydrogenation of alkynes to alkenes without further reduction to the corresponding alkanes is of considerable interest in the areas of polymer synthesis and fine chemicals. $^{144}$ In this context, IL microphase was used for enhancing selectivity of metal nanoparticles supported on a MOF $\left(\mathrm{Pd} / \mathrm{IL} / \mathrm{Cu}_{3}(\mathrm{BTC})_{2}\right) .{ }^{52}$ It is believed that IL microphase provides an adequate environment for stabilizing metal nanoparticles. Typically, the precursors of $\mathrm{Cu}_{3}(\mathrm{BTC})_{2}$ and Pd were loaded into the IL 1,1,3,3-tetramethylguanidinium trifluoroacetate (TMGT), followed by reduction, affording $\mathrm{Pd} / \mathrm{IL} / \mathrm{Cu}_{3}(\mathrm{BTC})_{2}$. FT-IR spectra showed the coordination of carboxylate groups of BTC to $\mathrm{Cu}(\mathrm{II})$ ions. The powder XRD pattern of the assynthesized $\mathrm{Pd} / \mathrm{IL} / \mathrm{Cu}_{3}(\mathrm{BTC})_{2}$ is in agreement with the MOF formation. ${ }^{145}$ Inductively coupled plasma atomic emission spectroscopy (ICP-AES) showed $1.27 \mathrm{wt} \%$ of $\mathrm{Pd}$ in $\mathrm{Pd} / \mathrm{IL} / \mathrm{Cu}_{3}(\mathrm{BTC})_{2}$. Furthermore, the IL content in $\mathrm{Pd} / \mathrm{IL} / \mathrm{Cu}_{3}(\mathrm{BTC})_{2}$ was $13.2 \mathrm{wt} \%$ as evidenced from TGA analysis. HRTEM images showed the existence of Pd NPs with an average size around $2 \mathrm{~nm}$. Nitrogen adsorption-desorption analysis revealed that $\mathrm{Pd} / \mathrm{IL} / \mathrm{Cu}_{3}(\mathrm{BTC})_{2}$ possesses BET surface area of $708 \mathrm{~m}^{2} \mathrm{~g}^{-1}$ while, the parent $\mathrm{Cu}_{3}(\mathrm{BTC})_{2}$ has a higher surface area of $1026 \mathrm{~m}^{2} \mathrm{~g}^{-1}$. This surface area decrease should be a reflection of the incorporation of IL and Pd NPs into the 
micropores of $\mathrm{Cu}_{3}(\mathrm{BTC})_{2}$. Phenylacetylene was completely converted to styrene upon hydrogenation (Figure 6) catalyzed by $\mathrm{Pd} / \mathrm{IL} / \mathrm{Cu}_{3}(\mathrm{BTC})_{2}$ with very high selectivity with a TOF value of $2287 \mathrm{~h}^{-1}$. Under identical conditions, $\mathrm{Pd} / \mathrm{Cu}_{3}(\mathrm{BTC})_{2}$ required more than twenty times longer time to reach $83 \%$ conversion with $99 \%$ selectivity to styrene $\left(99 \mathrm{~h}^{-1}\right)$. This activity difference was attributed to the fact that the Pd NPs undergo aggregation in the absence of IL. Comparison of the catalytic activity of $\mathrm{Pd} / \mathrm{IL} / \mathrm{Cu}_{3}(\mathrm{BTC})_{2}$ with $\mathrm{Pd} / \mathrm{C}$ and other reported catalysts $^{146}$ revealed that $\mathrm{Pd} / \mathrm{IL} / \mathrm{Cu}_{3}(\mathrm{BTC})_{2}$ shows much better performance in terms of conversion and selectivity under the same reaction conditions. It has been widely reported that $\mathrm{N}$ containing compounds can be used to modify the support to improve the selectivity of the catalyst for alkyne hydrogenation to alkenes. ${ }^{147-149}$ Hot filtration test proved the heterogeneity of the reaction. The catalyst was reused for four cycles with no significant decay in its activity. The TEM image of the five times used catalyst shows no evidence of particle aggregation and no major difference was seen in the fresh and recovered catalyst by powder XRD. ICP-AES analysis indicated Pd loading of the recovered catalyst was $1.23 \mathrm{wt} \%$, which is close to the loading of the fresh material (1.27 wt\%). Therefore, all the available characterization data indicate a remarkable stability of $\mathrm{Pd} / \mathrm{IL} / \mathrm{Cu}_{3}(\mathrm{BTC})_{2}$ under the reaction conditions.

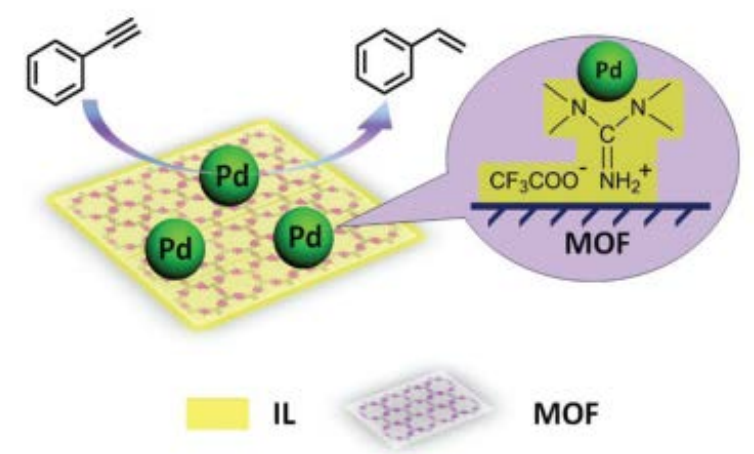

Figure 6. Synthesis of $\mathrm{Pd} / \mathrm{IL} / \mathrm{Cu}_{3}(\mathrm{BTC})_{2}$ catalyst and its catalytic activity for the selective partial hydrogenation of phenylacetylene. Reproduced from ref ${ }^{52}$ with the permission from Royal Society of Chemistry.

3.5 Hydrocarboxylation of Cyclohexane 
A Cu(II)-MOF was synthesised with 4-((4-([2,2':6',2'’-terpyridin]-4'yl)benzyl)oxy)benzoate] (Figure 7) as a flexible bifunctionalized terpyridine ligand. ${ }^{53}$ The catalytic activity of this terpyridinyl-containing Cu-MOF was examined in the low-temperature hydrocarboxylation of cyclohexane with carbon monoxide, water and potassium peroxodisulfate in water/acetonitrile or water/IL medium. The IL used was 1-butyl-1methylpyrrolidinium bis(trifluoromethanesulfonyl)imide, [BMPyr] [NTf 2 (Scheme 15) due to its stability towards CO. This reaction converts cyclohexane into the corresponding cyclohexanecarboxylic acid (Scheme 16). A noticeable advantage of this method is the use of aqueous medium and an IL, under mild conditions, without the need of an acid, such as trifluoroacetic acid that becomes waste in the reaction workup. In water/acetonitrile medium (3:3), the catalyst showed $33.4 \%$ yield of cyclohexanecarboxylic acid with a TON value of 44.5. On the other hand, the catalyst afforded $35.9 \%$ yield of the product with a TON value being 45.1 in water/ [BMPyr][NTf 2 ] medium (3:3), showing a slight improvement. Control experiments revealed that the solvent composition was an important parameter in the hydrocarboxylation of cycloalkanes, which does not proceed either in pure water, only in acetonitrile, or in the absence of both (only in cyclohexane). Furthermore, the reaction did not also proceed in pure IL. The activity and selectivity of the catalyst was maintained for four consecutive cycles, but, however, the activity dropped by $16 \%$ and $35 \%$ in the $5^{\text {th }}$ and $6^{\text {th }}$ cycle, respectively. Powder XRD of the four times reused catalyst did not show any change in the structural integrity of the Cu-MOF compared to the fresh catalyst. No leaching of Cu was also detected under the present experimental conditions.

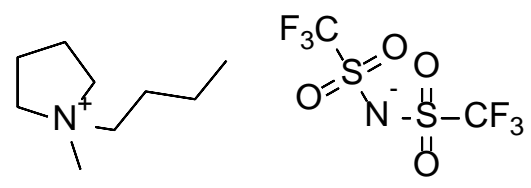

Scheme 15. Structure of 1-butylmethylpyrrolidium bis(trifluoromethanesulfonyl)imide, [BMPyr][NTf 2 . 


$$
\square+\mathrm{CO}+\mathrm{H}_{2} \mathrm{O}+\mathrm{S}_{2} \mathrm{O}_{8}{ }^{2-} \stackrel{\mathrm{Cu}-\mathrm{MOF}}{\longrightarrow}
$$

Scheme 16. Hydrocarboxylation of cyclohexane to cyclohexanecarboxylic acid promoted by $\mathrm{Cu}-\mathrm{MOF}$.
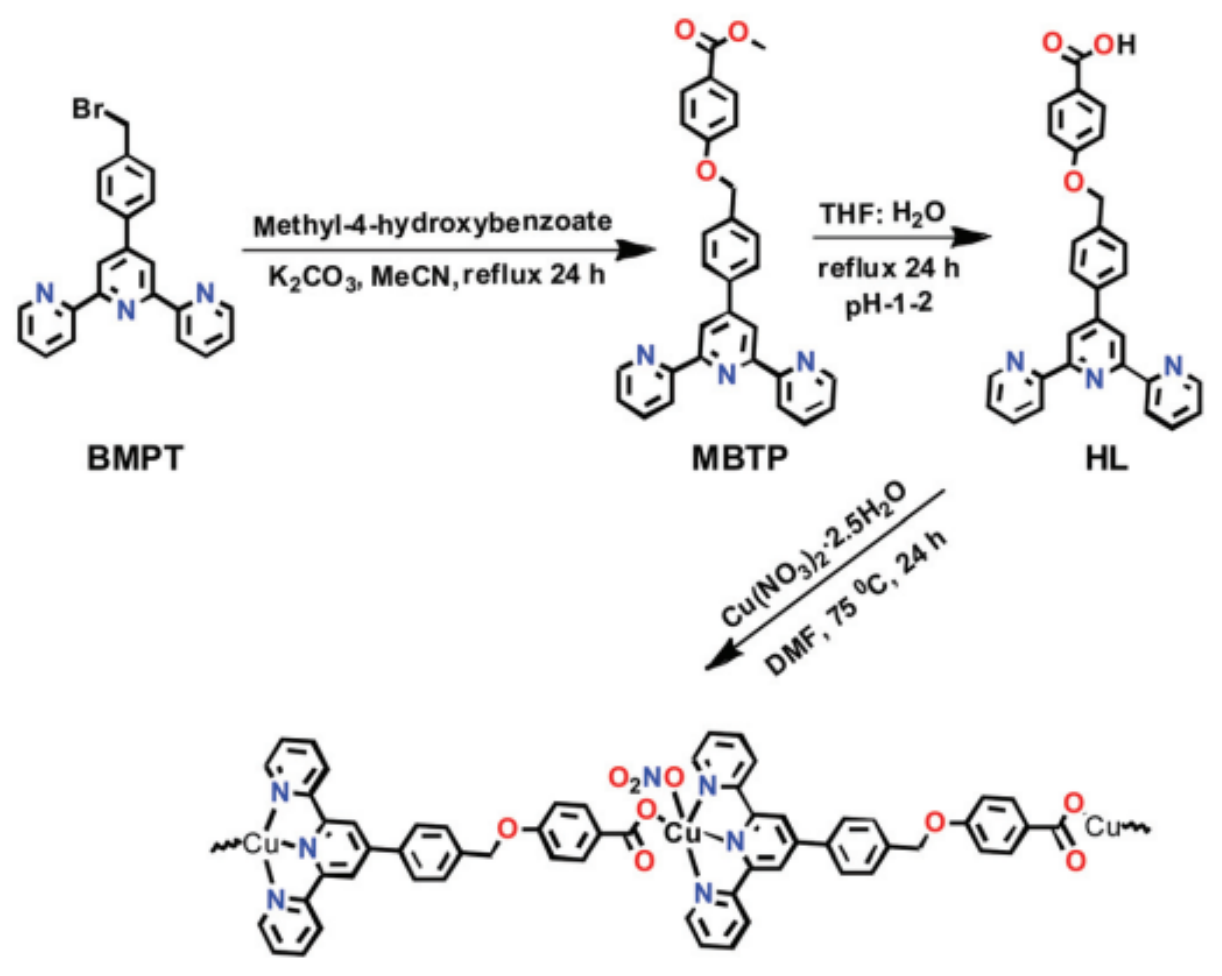

1

Figure 7. Synthesis of HL and formation of the corresponding MOF by reaction with copper nitrate. Reproduced from ref ${ }^{53}$ with the permission from Royal Society of Chemistry.

\subsection{Azide-Alkyne Cycloaddition}

Recently, CuI was loaded on UiO-67-type MOF, namely UiO-67-IM, to obtain CuI@UiO-67-IM. UiO-67-IM was already decorated with n-pentadecyl units attached to imidazolium salt via direct ligand functionalization (Figure 8) before adsorption of CuI. ${ }^{54}$ Powder XRD patterns of UiO-67-IM and CuI@UiO-67-IM indicated that both samples were highly crystalline, and their structure was identical to that of pristine UiO-67. Thus, the preembedded imidazolium salt with long alkyl linear chain and subsequent coincorporation of CuI 
did not affect the framework of UiO-67. The BET surface area of CuI@UiO-67-IM was (45.1 $\mathrm{m}^{2} / \mathrm{g}$ ) which is significantly lower than that of UiO-67 (2113 m²/g). The activity of CuI@UiO67-IM was examined in the $\mathrm{Cu}(\mathrm{I})$-catalyzed azide-alkyne cycloaddition between phenylacetylene and benzyl azide to achieve $89 \%$ conversion with $99 \%$ selectivity of the expected 1, 4-disubstituted triazole (Scheme 17) at $80{ }^{\circ} \mathrm{C}$ in water under air atmosphere for $2 \mathrm{~h}$. Also, the reaction was heterogeneous in nature since there was no further conversion after removal of the solid catalyst from the reaction mixture. The recyclability of CuI@UiO-67-IM was studied in one-pot, two-step domino reaction based on 4-nitrobenzyl bromide, $\mathrm{NaN}_{3}$ and phenylacetylene. The catalyst showed conversions of 94 to $90 \%$ with $>99.9 \%$ selectivity within five catalytic runs. In addition, powder XRD patterns and SEM images of CuI@UiO67-IM after five catalytic runs confirmed that the structural integrity and morphology of CuI@UiO-67-IM were retained after the reaction. Furthermore, no valence change was observed for copper species as determined by XPS after five catalytic cycles. However, there was a leaching of CuI to the level of $10.7 \%$ of the initial CuI amount as determined by ICPAES. 
<smiles>COC(=O)c1ccc(-c2ccc(C(=O)OC)cc2Cn2ccnc2)cc1</smiles>

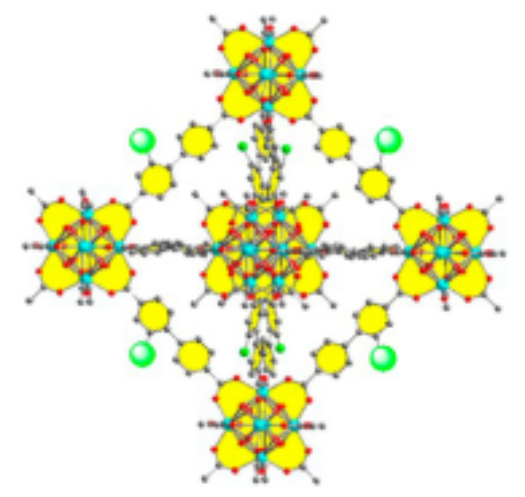

UiO-67-IM (1)

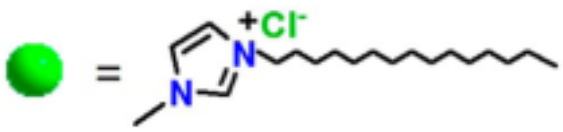

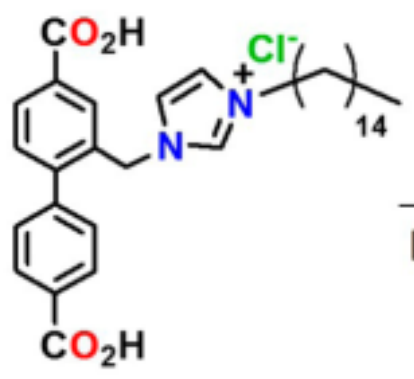

$\underset{\mathrm{HOAc}, 120^{\circ} \mathrm{C}}{\stackrel{\mathrm{ZrCl}_{4}, \mathrm{DMF}}{\longrightarrow}}$

Cul, $\mathrm{CH}_{3} \mathrm{CN}$

r.t.

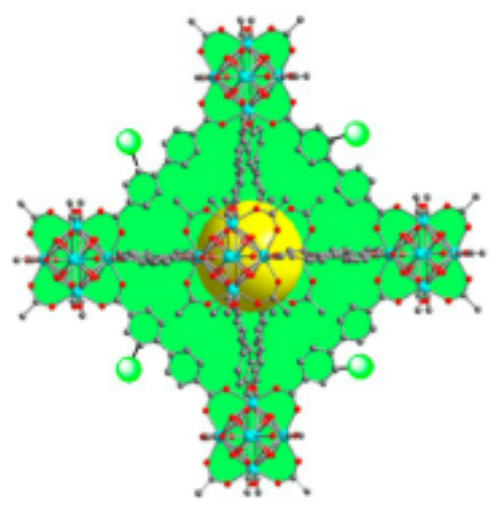

Cul@UiO-67-IM (2)

$=$ Cul

Figure 8. Synthesis of L, UiO-67-IM and CuI@UiO-67-IM. Reproduced from ref ${ }^{54}$ with the permission from American Chemical Society.<smiles>[M]CCCO[GeH3]</smiles><smiles>c1ccc(Cn2cc(-c3ccccc3)nn2)cc1</smiles><smiles>c1ccc(Cn2nncc2-c2ccccc2)cc1</smiles>

1,4-isomer

Scheme 17.CuI@UiO-67-IM catalyzed cycloaddition reaction between benzyl azide and phenylacetylene.

\subsection{Coupling of $\mathrm{CO}_{2}$ with Epoxide}

ILs have been among the preferred solvents for $\mathrm{CO}_{2}$ reactions, including $\mathrm{CO}_{2}$ insertion to epoxides. The advantages of IL in these reactions derive not only from the solubility that $\mathrm{CO}_{2}$ shows in these liquids, but also that halides as counter anions of the IL act as a nucleophile 
activating the $\mathrm{C}$ atom of $\mathrm{CO}_{2}$, favouring the distortion of this reagent from the linearity. Not surprisingly, ILs embedded within MOFs have also been used as catalysts for this reaction type.

A strategy was developed to combine together coordinatively unsaturated metal sites based MOFs with functional IL to form heterogeneous bifunctional catalysts (Figure 9) and its activity was tested in the cycloaddition of $\mathrm{CO}_{2}$ with epoxides under mild conditions in the absence of co-catalyst. In this context, two bifunctional heterogeneous catalysts incorporating IL, MIL-101(Cr)-X(n-Bu) ${ }_{3} \mathrm{Br}(\mathrm{X}: \mathrm{N}$ or $\mathrm{P})$ were synthesised with coordinatively unsaturated sites as Lewis acid centres and embedding ILs as additional active sites. ${ }^{55}$ Characterization data by powder XRD and IR spectroscopy indicated the formation of MIL-101-NH 2 and its modification by ILs. MIL-101-N(n-Bu) ${ }_{3} \mathrm{Br}$ and MIL-101-P(n-Bu) ${ }_{3} \mathrm{Br}$ converted $\mathrm{CO}_{2}$ and propylene oxide into propylene carbonate (PC, Scheme 18) with 99.1 (110.1 TON) and 98.6 \% (109.6 TON) yields, respectively. Control experiments using mixtures of pure components MIL-101- $\mathrm{NH}_{2}$, $(\mathrm{nBu})_{4} \mathrm{NBr}$ or $(\mathrm{n}-\mathrm{Bu})_{4} \mathrm{PBr}$ showed comparatively lower yield of PC under the same conditions, which can be rationalized due to the existence of only monofunctional catalytic sites in these materials. Furthermore, although the physical mixtures of MIL-101-NH2 with (n-Bu) ${ }_{4} \mathrm{NBr}$ and MIL-101- $\mathrm{NH}_{2}$ with (n-Bu) $)_{4} \mathrm{PBr}$ exhibited 98.4 and $97.9 \%$ yields, respectively, which are similar values to those achieved by MIL-101-N(n-Bu) ${ }_{3} \mathrm{Br}$ and MIL101-P(n-Bu $)_{3} \mathrm{Br}$, the advantage of the IL-functionalized MOFs was the lack of activity decay after three cycles, while the catalysts consisting in the physical mixture of IL and MOF showed a dramatic decrease in the yield in second cycle. In one case, ICP analysis revealed the presence of $0.012 \mathrm{mg}$ of phosphorus in solution. IR spectroscopy and powder XRD patterns for the fresh and recycled MIL-101(Cr)-X-(n-Bu) 3 Br catalysts did not show appreciable changes, thus, supporting the stability of the material under the experimental reaction conditions. Furthermore, the advantage of these bifunctional MOF catalysts was confirmed by comparing their activity with some benchmark MOFs ${ }^{150-154}$ under the same reaction conditions. As shown 
in Figure 10, MIL-101-X-(n-Bu) ${ }_{3} \mathrm{Br}$ showed the highest yield to PC (> 98\% conversion). In contrast, other MOFs exhibit only a very low conversion with 2.5, 5.2, 5.4, 20.9 and $23.2 \%$ for MOF-5, IRMOF-3, HKUST-1, MIL-101 and Mg-MOF-74, respectively. This enhanced catalytic activity of MIL-101(Cr)-X-(n-Bu) $)_{3} \mathrm{Br}$ was believed to be due to the synergetic catalytic roles of Lewis acid sites and $\mathrm{Br}^{-}$of ILs as nucleophile. These experiments clearly demonstrate that bifunctional catalysts offer enhanced activity compared to mono functional catalysts.

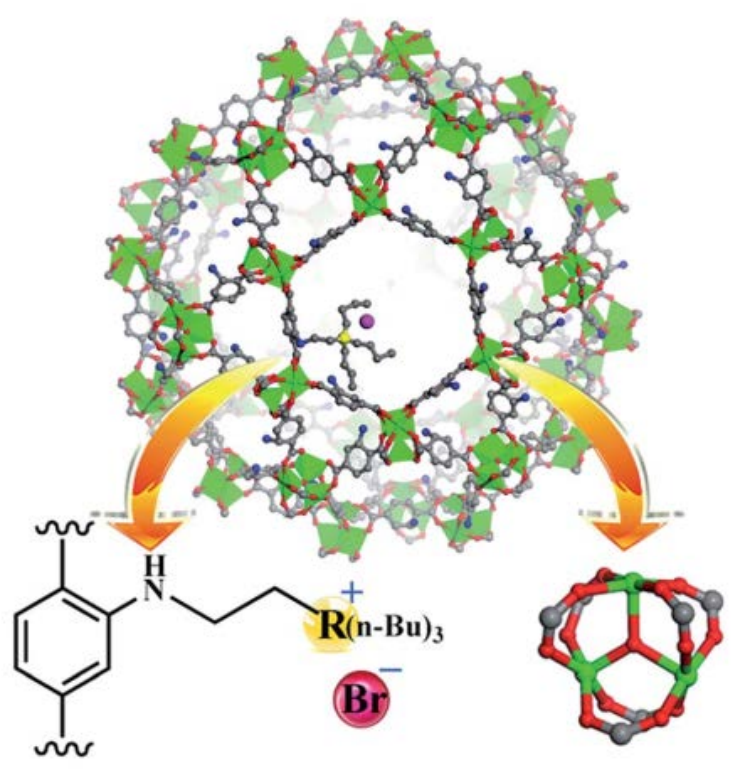

Figure 9. Structure of MIL-101(Cr)-X-(n-Bu) ${ }_{3} \mathrm{Br}$ (Cr: green, C: gray, O: red, N: blue, Br: amaranth, and $\mathrm{R}=\mathrm{N}$ or $\mathrm{P}$ ). Reproduced from ref ${ }^{55}$ with the permission from Royal Society of Chemistry. 


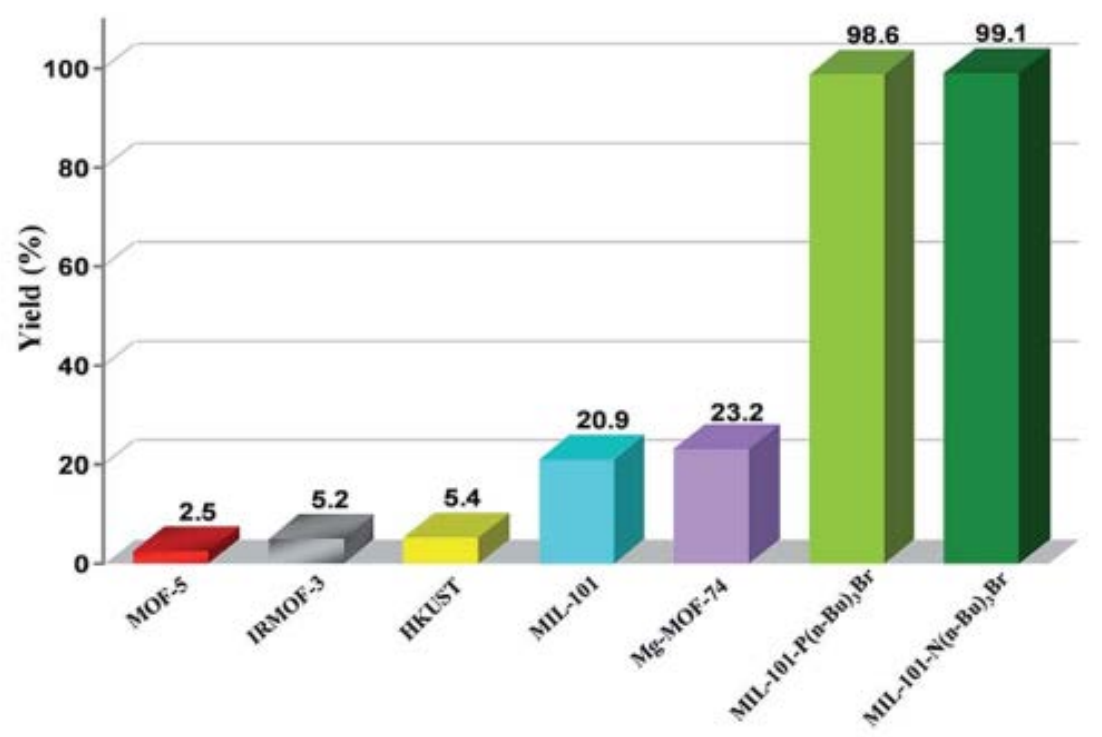

Figure 10. Comparison of PC yield in the cycloaddition $\mathrm{CO}_{2}$ to propylene oxide and catalyzed by bifunctional MIL-101(Cr)-X-(n-Bu) ${ }_{3} \mathrm{Br}$ compared to other MOFs. Reproduced from ref ${ }^{55}$ with the permission from Royal Society of Chemistry.

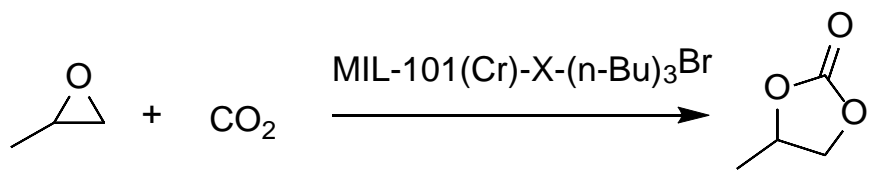

Scheme 18. Reaction of propylene oxide with $\mathrm{CO}_{2}$ catalyzed by MIL-101(Cr)-X-(n-Bu) 3 Br.

A new imidazolium-based UiO-67 type MOF (UiO-67-IL) was synthesised using ligand functionalization (Figure 11). ${ }^{56}$ The catalytic activity of UiO-67-IL was studied in the cycloaddition of $\mathrm{CO}_{2}$ to epichlorohydrin (Scheme 19) with or without TBAB as co-catalyst under 1 atm of $\mathrm{CO}_{2}$. Powder XRD pattern indicates that UiO-67-IL is highly crystalline and its structure is similar to that of pristine UiO-67. The use of UiO-67-IL afforded $99 \%$ yield (45.08 $\mathrm{h}^{-1}$ TOF) of the desired product with TBAB as co-catalyst at $90{ }^{\circ} \mathrm{C}$, while the same catalyst required under identical conditions much longer time to reach $88 \%$ yield (13.66 h ${ }^{-1}$ TOF) without TBAB. On the other hand, no catalytic activity was observed with UiO-67 in the absence of IL even at $90{ }^{\circ} \mathrm{C}(<3 \%$ yield), but, however, the carbonate yield increased to $8 \%$ yield for longer time. In contrast, UiO-67-IL with imidazolium moieties afforded $95 \%$ yield 
under similar conditions. On the other hand, the UiO-67-TBAB catalytic system showed $98 \%$ yield, whereas UiO-67-IL gave $99 \%$ yield in less than half the time for epichlorohydrin $\mathrm{CO}_{2}$ cycloaddition. These data clearly illustrate the pivotal role of embedded imidazolium group in UiO-67-IL catalyzing this reaction. No leaching of active sites was detected under this experimental condition. Also, the catalyst was reused for five catalytic runs. Powder XRD patterns of the fresh and five times reused catalyst showed that the structural integrity is maintained.

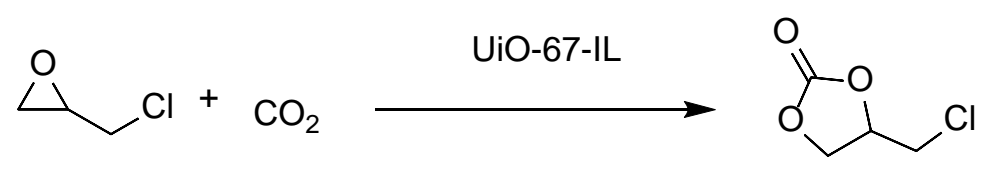

Scheme 19. Cycloaddition of $\mathrm{CO}_{2}$ to epichlorohydrin catalyzed by UiO-67-IL.

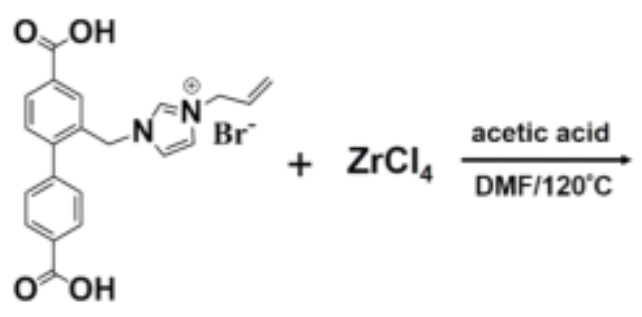

L

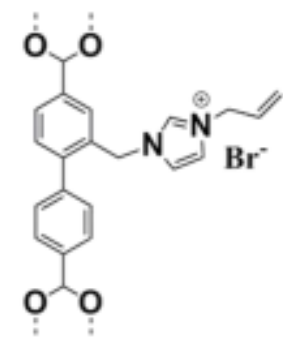

UiO-67-IL (1)

Figure 11. Synthesis of UiO-67-IL by using an IL-functionalized biphenyldicarboxylic acid as linker. Reproduced from ref ${ }^{56}$ with the permission from American Chemical Society.

Recently, the covalent post functionalization of zeolitic imidazolate framework-90 (ZIF-90) with a pyridinium based IL was performed to obtain IL-supported ZIF-90 (IL-ZIF90) and its activity is examined in the solvent-less cycloaddition of epoxides with $\mathrm{CO}_{2}{ }^{57} 2$ Imidazolecarboxaldehyde (ICA) showed $19 \%$ conversion while aminopyridinium iodide (AMPyI) afforded $44 \%$ conversion with $100 \%$ selectivity, whereas the combined physical mixture of all the starting materials (zinc nitrate, ICA and AmPyI) employed for the synthesis of IL-ZIF-90 resulted in slightly higher conversion of 55\% under identical conditions. On the 
other hand, the use of ZIF-90 as catalyst resulted in 51\% conversion which is slightly lower to the combination of ZIF-90 and AmPyI (65 \% conversion). Interestingly, the IL-ZIF-90 exhibited $97 \%$ conversion with $98 \%$ selectivity under identical conditions. These data clearly demonstrate the advantage of post functionalization in MOFs enhancing the activity of ILs within the frameworks of MOFs. Among the various epoxides screened, propylene oxide afforded a maximum conversion of $97 \%$, whereas cyclohexene oxide showed $9 \%$ conversion under identical conditions. This lower activity was due to the steric hindrance caused by the cyclohexene ring. IL-ZIF-90 was reused for four successive runs without any significant decay in its activity. Powder XRD of the fresh catalyst did not show any noticeable changes in the four times reused catalyst.

\section{Hierarchical Porous MOFs for Catalysis}

In recent years, the development of hierarchically porous MOFs with controlled micro/mesoporosity has received considerable interest due to their better performance in practical industrial processes such as adsorption, catalysis and chemical sensing. ${ }^{155}$ Also, achieving hierarchical structures in heterogeneous solids are of great interest as they feature high pore volumes, large surface areas and larger pore sizes. Very recently, a new method, namely “linker labilization”, has been developed to increase the MOF porosity and pore size, thus leading to hierarchical-pore architectures. $^{156}$ In this method, microporous MOFs were initially synthesized using robust metal nodes and pro-labile linkers. Then, the mesopores were subsequently created as crystal defects through the splitting of a pro-labile-linker followed by the removal of the linker fragments by acid treatment. It was facilely shown that the linker labilization method can create controllable hierarchical porous structures in stable MOFs, which allows the diffusion and adsorption of cytochrome c in an efficient manner. 
Recently, a facile route has been reported for the preparation of carbon nitride (CN) foams as structural templates with micrometer-sized pores with the nitrogen content of 25.6 wt $\%$ by the fast carbonization of melamine foam (Figure 12). The nitrogen functionalities of CN foam provide the feasibility of chemical anchoring and growth of ZIF-8 crystals, thus leading to the development of hierarchical porous MOFs. ${ }^{157}$ Furthermore, the growth of ZIF-8 crystals also renders $\mathrm{CN}$ foam which is hydrophilic in nature is converted as a highly hydrophobic, thus creating an effective shield for the MOF crystals against water. As a result, the introduction of ZIF-8 crystals onto the CN foam provides selective absorption of oils up to $58 \mathrm{wt} \%$ from water/oil mixtures and also facilitates the highly efficient conversion of $\mathrm{CO}_{2}$ to 3-chloropropene carbonate in a quantitative yield with selectivity. In contrast, the use of ZIF8-based catalysts for this reaction showed low product selectivity to 3-chloropropene carbonate (52\%) and also produce by-products including diols (23.7\%) and dimer (24.3\%). ${ }^{158}$ Also, ZIF8 catalysts showed significant losses in their catalytic activity after the first cycle whereas ZIF8/CN foam retained high selectivity and conversion up to fourth cycles. Hence, this approach could be extended to those MOFs that have poor stability in water.

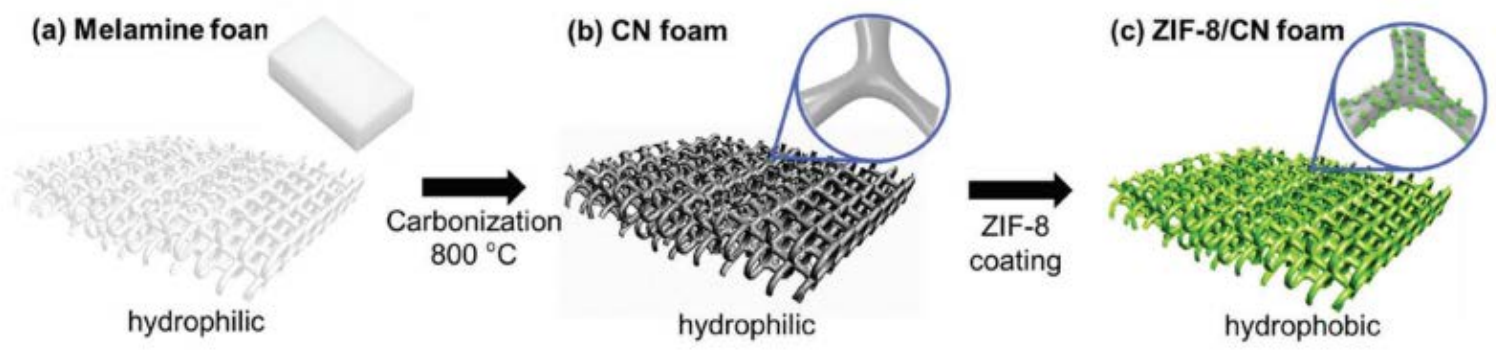

Figure 12. Schematic representation of the synthesis of ZIF-8/CN foam with hierarchical porosity. Reproduced from ref ${ }^{157}$ with the permission from Wiley.

The ability of MOFs to gelate under specific experimental conditions provides further opportunities in the preparation and shaping of hierarchically porous MOF monoliths, which could be used in catalysis and adsorption applications. Recently, xero- or aerogel monoliths 
consisting of solely nanoparticles of several prototypical $\mathrm{Zr}^{4+}$-based MOFs such as UiO-66-X $\left(\mathrm{X}=\mathrm{H}, \mathrm{NH}_{2}, \mathrm{NO}_{2},(\mathrm{OH})_{2}\right), \mathrm{UiO}-67, \mathrm{MOF}-801, \mathrm{MOF}-808$ and NU-1000 have been reported. ${ }^{159}$ Furthermore, UiO-66 gels were shaped into monolithic spheres of $600 \mathrm{~mm}$ diameter using an oil drop method, thus creating different materials which could find applications in packedbed catalytic or adsorptive applications, where hierarchical pore systems can greatly weaken mass transfer limitations.

A pyridyl-decorated MOF-505 analogue $\left[\mathrm{Cu}_{2}(\mathrm{~L})\left(\mathrm{H}_{2} \mathrm{O}\right)_{2}\right] \cdot \mathrm{Gx}(\mathrm{L}=5,5$ '-(pyridine-2,5diyl)-diisophthalic acid, G: solvent molecule) has been reported with rare hierarchical mesoand microporosity with exposed unsaturated $\mathrm{Cu}^{\mathrm{II}}$ sites and Lewis basic pyridyl sites. The catalytic activity of this catalyst was reported in the cyanosilylation reaction under solvent-free conditions. ${ }^{160}$ The dehydrated catalyst showed $99 \%$ conversion in the cyanosilylation of benzaldehyde at $40{ }^{\circ} \mathrm{C}$. The catalytic activity of this hierarchical MOF is comparable to other $\mathrm{Cu}$ or $\mathrm{Zr}$-based MOFs ${ }^{161-163}$ in the cyanosilylation of benzaldehyde but is considerably higher than other catalysts like $\left.\mathrm{Cu}_{3}(\mathrm{BTC})\right)_{2}(50-60 \%),{ }^{126} \mathrm{Cd}-\mathrm{MOF}(77 \%),{ }^{164}$ and Ln-based MOFs (5 mol\%, 77.8-94.8 \%, $\left.50{ }^{\circ} \mathrm{C}\right) .{ }^{165}$ This enhanced activity is due to the presence of a high density of the exposed metal sites and the improved molecular accessibility of the hierarchical mesoand microporosity. Catalysis was heterogeneous in nature and the MOF-505 was recycled up to five times with only a minor loss of activity.

In another precedent, a continuous phase transformation processing strategy has been applied for fabricating and shaping MOFs into shaped bodies and MOF foams that exhibit reversible transformation among these states. In this context, a cup-shaped reactor based on $\mathrm{Cu}-\mathrm{MOF}$ composite and hierarchically porous MOF foam were prepared and their activity was compared in the oxidation of diphenylmethane with TBHP as an oxidant. ${ }^{166}$ The use of cupshaped reactor afforded $76 \%$ conversion with $93 \%$ selectivity, while using porous foam enhances the conversion to $92 \%$ with $97 \%$ selectivity under identical conditions. 
Furthermore, quenched solid-state density functional theory calculation revealed that the foam possesses hierarchically distributed porosity with the pore size distribution consisting of both micro- and mesopores expanding up to $40 \mathrm{~nm}$. The hierarchically porous MOF foam was reused for three cycles with no significant decay in its activity.

Recently, a coordination replication strategy has been applied to construct hierarchical Cu-based MOF nanoarrays and their activity has been examined in the reduction of 4nitrophenol to 4-aminophenol using sodium borohydride as reducing agent. ${ }^{167}$ In this approach, $\mathrm{Cu}(\mathrm{OH})_{2}$ nanorods were the source for $\mathrm{Cu}$ that coordinate with a series of organic ligands to form different MOF crystals, and they also served as a 3 D substrate to support the growth of the Cu-based MOF (Figure 13). Hierarchical MOF nanoarrays exhibited enhanced activity in the reduction of 4-nitrophenol compared with those on MOF-2 nanoparticles and $\mathrm{Cu}(\mathrm{OH})_{2}$ nanorod arrays. This is due to the unique 3D hierarchical nanoarray architecture of the nanocomposite. There was no significant loss in activity for eight successive catalytic cycles thus showing the long term stability of hierarchical MOFs. 


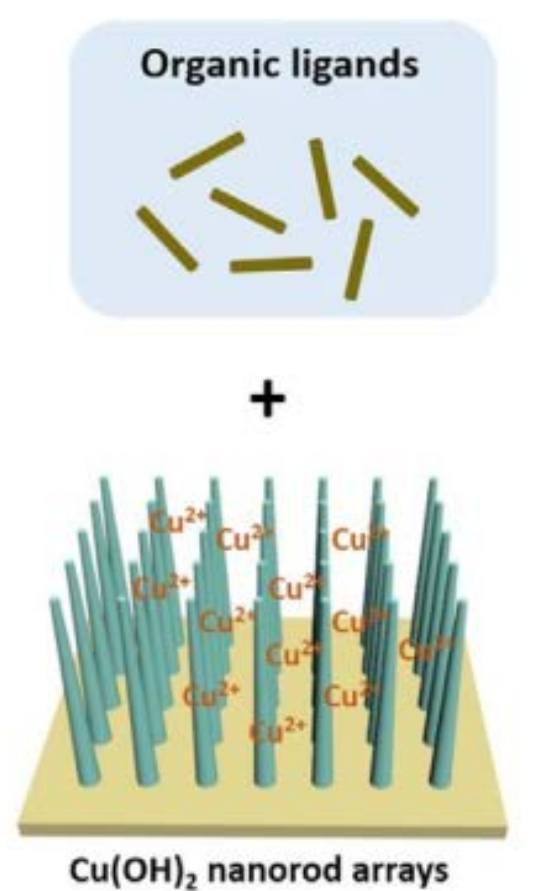

Figure

\section{Coordination replication}

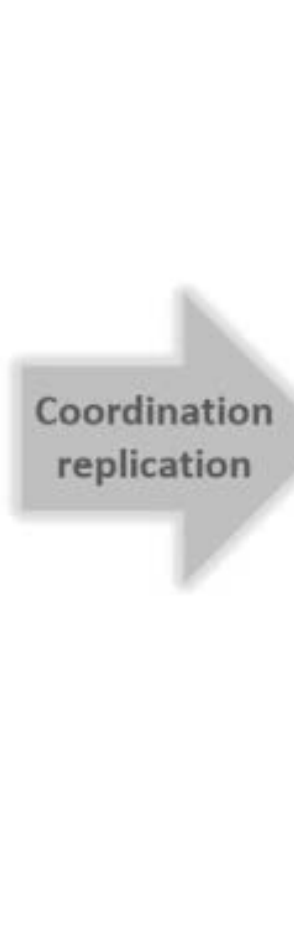

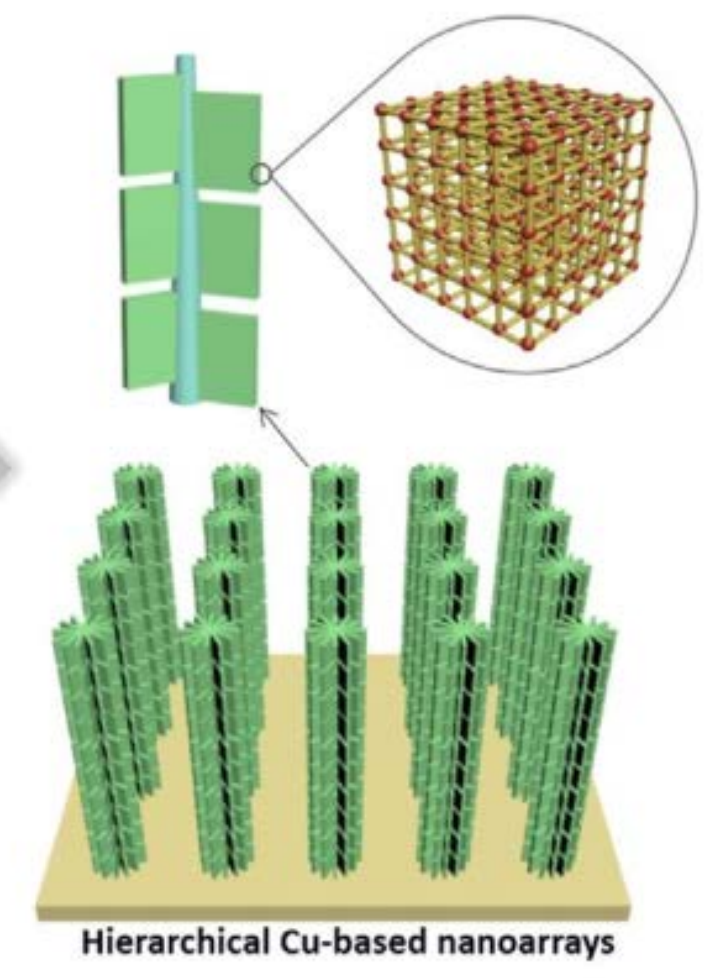

Cu-based MOF

nanoarrays by the coordination replication strategy. Reproduced from ref ${ }^{167}$ with the permission from Wiley.

Two hierarchically porous MOFs namely, NUS-6 (NUS: National University of Singapore) composed of either zirconium (Zr) or hafnium (Hf) clusters were synthesised using modulated hydrothermal method with high stability and strong Brönsted acidity. ${ }^{168}$ These MOFs exhibit BET surface areas of 550 and $530 \mathrm{~m}^{2} / \mathrm{g}$ for NUS-6(Zr) and NUS-6(Hf), respectively, and a hierarchically porous structure of coexisting micropores $(\sim 0.5, \sim 0.7$, and $\sim 1.4 \mathrm{~nm})$ and mesopores $(\sim 4.0 \mathrm{~nm})$ with dangling sulfonic acid groups. Structural analysis showed that the hierarchical porosity of NUS-6 derives from missing linkers and clusters of the parent UiO-66 framework. The activity of these MOFs was tested in the dehydration of fructose to 5-hydroxymethylfurfural (HMF), in which NUS-6(Hf) showed $98 \%$ yield which is superior than NUS-6(Zr) (84 \% yield) after $1 \mathrm{~h}$ at $100{ }^{\circ} \mathrm{C}$. This enhanced activity is due to the 
stronger Brönsted acidity contribution from $\mathrm{Hf}-\mu_{3}-\mathrm{OH}$ groups as well as the availability of smaller pore sizes to restrict undesirable reactions.

The above examples illustrate how the preparation of hierarchical MOFs with controlled micro-/mesoporosity and particle shapes can enhance the catalytic activity of these solids by facilitating the accessibility of substrates and reagents to the active sites. This particle shaping is of large importance in commercial catalysts used in industry, but is an aspect that is frequently ignored in most catalytic studies. Shaping MOF particles with an adequate morphology and ordering is a necessary step to be considered in any potential application of these materials as adsorbents and catalysts.

\section{Summary and Future Outlook}

It has been shown that MOFs are suitable solid catalysts also under conditions in which the presence of conventional organic solvents are avoided or when incorporating ILs that possess active sites. This includes solvent-free conditions using MOFs, but also other examples in where MOFs have been modified by adsorbing ILs, either embedded or attached to the MOF lattice. In the last case these ILs act not only modifying the polarity of the reaction, but also frequently by adding additional acid/basic sites that cooperate to the reaction mechanism, increasing the efficiency of the catalytic process. At the present, the number of studies are still limited, although there are several reaction types that have been sufficiently documented that can occur under these favourable green conditions in the absence of solvents such as condensations, silylations, selective partial hydrogenation of alkynes to alkenes and $\mathrm{CO}_{2}$ insertions. It will be important to expand the use of MOFs under solvent-free conditions to other reaction types, particularly to oxidations and couplings that are processes highly sensitive to the nature of solvent and that can generate high amounts of wastes, trying to delineate when solvents can be avoided and gain understanding on the reasons. 
One point that has not been in our opinion sufficiently addressed is MOF stability as catalyst under solvent-free conditions. It should be considered that the most robust MOFs have pore sizes of $1 \mathrm{~nm}$ or below and structure stability decreases as the pore size increases. Besides the lack of structural stability that is a general issue to be carefully addressed when using MOFs as catalysts, specific points that need to be investigated is how product adsorption affects to the catalytic activity and what the causes of deactivation under solvent-free conditions are. A general claim in most of the reports presented is the lack of the catalyst deactivation without paying attention to why product inhibition or byproduct formation do not seem to occur in these studies. In other words, all the solid catalysts should deactivate upon reuse and solvent-free conditions are specially favourable for poising due to the high concentration of products/byproducts and the absence of desorption by solvent molecules. It would be important to establish an in-depth study of the reasons why this deactivation process does not happen and the system appears stable deserve always.

Another important aspect that has been frequently neglected is particle shaping and structuring resulting in the formation of hierarchical MOF catalysts. The presence of porosity combining micro- and mesoporosity is known to facilitate mass transport and this result in an enhanced activity using heterogeneous catalysts. Examples have been presented showing that the flexibility in MOF synthesis allows to develop different strategies like linker labilization, gelation or synthesis on foams that have resulted in hierarchical MOFs.

Overall, the present review has shown that MOFs can contribute to the greenness of chemical processes not only by acting as efficient and selective solid catalysts, but also by avoiding the use of in some cases of volatile solvents without the penalty of fast deactivation. Similarly, decoration or coating of the internal pores of MOFs by IL units has shown that it is possible to combine the effects of molecular ILs with that of a solid catalyst with the minimum amount of IL and with advantages in terms of recovery of IL and workup of the reaction 
mixture. It has also been shown that the preparation of MOFs exhibiting a hierarchical structuring enhances their catalytic activity by facilitating the mass transport. Since MOF catalysis is under intense investigation, it can be predicted that additional efforts will be made to expand the use of MOFs as heterogeneous catalysis using the three commented toolsto other types of reactions.

\section{Acknowledgements}

AD thanks the University Grants Commission (UGC), New Delhi, for the award of an Assistant Professorship under its Faculty Recharge Programme. AD also thanks the Department of Science and Technology, India, for the financial support through Extra Mural Research Funding (EMR/2016/006500). Financial support by the Spanish Ministry of Economy and Competitiveness (Severo Ochoa and CTQ2015-69153-CO2-1) is gratefully acknowledged.

\section{References}

1. R. A. Sheldon, I. W. C. E. Arends and U. Hanefeld, Green Chemistry and Catalysis, 2007, doi:10.1002/9783527611003.

2. J. H. Clark, R. Luque and A. S. Matharu, Annual Rev. Chem. Biomol. Eng., 2012, 3, 183-207.

3. R. Cernansky, Nature, 2015, 519 379-380.

4. K. Sanderson, Nature, 2011, 469 18-20.

5. M. Poliakoff and P. Licence, Nature, 2007, 450 810-812.

6. J. H. Clark, Green Chem., 1999, 1, 1-8.

7. J. Liu, L. Chen, H. Cui, J. Zhang, L. Zhang and C.-Y. Su, Chem. Soc. Rev., 2014, 43, 6011-6061.

8. J. Gascon, A. Corma, F. Kapteijn and F. X. Llabres i Xamena, ACS Catal., 2014, 4, 361-378.

9. A. Dhakshinamoorthy, A. M. Asiri and H. Garcia, Chem. Soc. Rev., 2015, 44, 1922-1947.

10. N. Stock and S. Biswas, Chem. Rev., 2012, 112 933-969.

11. W. Lu, Z. Wei, Z.-Y. Gu, T.-F. Liu, J. Park, J. Park, J. Tian, M. Zhang, Q. Zhang, T. Gentle III, M. Bosch and H.-C. Zhou, Chem. Soc. Rev., 2014, 43, 5561-5593.

12. M. L. Foo, R. Matsuda and S. Kitagawa, Chem. Mater., 2014, 26, 310-322.

13. J. Jiang and O. M. Yaghi, Chem. Rev., 2015, 115 6966-6997.

14. L. Zhu, X.-Q. Liu, H. L. Jiang and L.-B. Sun, Chem. Rev., 2017, 117 8129-8176.

15. A. Dhakshinamoorthy and H. Garcia, Chem. Soc. Rev., 2012, 41, 5262-5284.

16. A. Dhakshinamoorthy, M. Alvaro and H. Garcia, Catal. Sci. Technol., 2011, 1, 856-867.

17. A. Dhakshinamoorthy, A. M. Asiri and H. Garcia, Chem. Eur. J. , 2016, 22, 8012-8024.

18. A. Dhakshinamoorthy, M. Opanasenko, J. Cejka and H. Garcia, Adv. Synth. Catal., 2013, 355, 247-268. 
19. A. H. Chughtai, N. Ahmad, H. A. Younus, A. Laypkov and F. Verpoort, Chem. Soc. Rev., 2015, 44, 6804-6849.

20. D. Zhao, M. Wu, Y. Kou and E. Min, Catal. Today, 2002, 74, 157-189.

21. T. Welton, Coord. Chem. Rev., 2004, 248, 2459-2477.

22. V. I. Pârvulescu and C. Hardacre, Chem. Rev., 2007, 107 2615-2665.

23. K. Fujie and H. Kitagawa, Coord. Chem. Rev., 2016, 307 382-390.

24. A. Bhunia, S. Dey, J. M. Moreno, U. Diaz, P. Concepcion, K. V. Hecke, C. Janiak and P. Van Der Voort, Chem. Commun., 2016, 52, 1401-1404.

25. L. M. Aguirre-Díaz, M. Iglesias, N. Snejko, E. Gutiérrez-Puebla and M. Ángeles Monge, CrystEngComm, 2013, 15, 9562-9571.

26. L. M. Aguirre-Diaz, M. Iglesias, N. Snejko, E. Gutierrez-Puebla and M. Angeles Monge, RSC Adv., 2015, 5, 7058-7065.

27. L.-J. Zhang, C.-Y. Han, Q.-Q. Dang, Y.-H. Wang and X.-M. Zhang, RSC Adv., 2015, 5, 2429324298.

28. R. F. D'Vries, M. Iglesias, N. Snejko, E. Gutierrez-Puebla and M. A. Monge, Inorg. Chem., 2012, 51, 11349-11355.

29. W. Jiang, J. Yang, Y.-Y. Liu, S.-Y. Song and J.-F. Ma, Inorg. Chem. , 2017, 56, 3036-3043.

30. F. Liu, Y. Xu, L. Zhao, L. Zhang, W. Guo, R. Wang and D. Sun, J. Mater. Chem. A, 2015, 3, 2154521552.

31. M. Thimmaiah, P. Li, S. Regati, B. L. Chen and J. C. G. Zhao, Tetrahedron Lett., 2012, 53, 48704872.

32. S. Rostamnia and A. Morsali, RSC Adv., 2014, 4, 10514-10518.

33. S. Rostamnia and H. Xin, Appl. Organomet. Chem., 2014, 28, 359-363.

34. M. Saikia, D. Bhuyan and L. Saikia, Appl.Catal. A: Gen., 2015, 505 501-506.

35. S. Beheshti and A. Morsali, RSC Adv., 2014, 4, 37036-37040.

36. N. Anbu and A. Dhakshinamoorthy, J. Colloid Interf. Sci., 2017, 494 282-289.

37. S. Beheshti and A. Morsali, RSC Adv., 2014, 4, 41825-41830.

38. P. Li, S. Regati, H. Huang, H. D. Arman, J. C.-G. Zhao and B. Chen, Inorg. Chem. Front., 2015, 2, 42-46.

39. P. Li, S. Regati, H.-C. Huang, H. D. Arman, B.-L. Chen and J. C.-G. Zhao, Chinese Chem. Lett., 2015, 26 6-10.

40. O. V. Zalomaeva, A. M. Chibiryaev, K. A. Kovalenko, O. A. Kholdeeva, B. S. Balzhinimaev and V. P. Fedin, J. Catal., 2013, 298 179-185.

41. X. Zhou, Y. Zhang, X. Yang, L. Zhao and G. Wang, J. Mol. Catal. A: Chem. , 2012, 361-362 12-16.

42. R. Babu, R. Roshan, A. Cherian Kathalikkattil, D. W. Kim and D.-W. Park, ACS Appl. Mater. Interfaces, 2016, 8, 33723-33731.

43. Q. x. Luo, X.-D. Song, M. Ji, S.-E. Park, C. Hao and Y.-q. Li, Appl. Catal. A: Gen., 2014, 478 8190.

44. Q.-X. Luo, M. Ji, S.-E. Park, C. Hao and Y.-Q. Li, RSC Adv., 2016, 6, 33048-33054.

45. J. Wu, Y. Gao, W. Zhang, Y. Tan, A. Tang, Y. Men and B. Tang, Appl. Organometal. Chem. , 2015, 29, 96-100.

46. S. Abednatanzi, K. Leus, P. G. Derakhshandeh, F. Nahra, K. De Keukeleere, K. Van Hecke, I. Van Driessche, A. Abbasi, S. P. Nolan and P. Van Der Voort, Catal. Sci. Technol., 2017, 7, 1478-1487.

47. S. Abednatanzi, A. Abbasi and M. Masteri-Farahani, Catal. Commun., 2017, 96, 6-10.

48. Z. Wu, C. Chen, H. Wan, L. Wang, Z. Li, ;, B. Li, Q. Guo and G. Guan, Energy Fuels, 2016, 30, 10739-10746.

49. H. Wan, C. Chen, Z. Wu, Y. Que, Y. Feng, W. Wang, L. Wang, G. Guan and X. Liu, ChemCatChem 2015, 7, 441-449.

50. H. M. A. Hassan, M. A. Betiha, S. K. Mohamed, E. A. El-Sharkawy and E. A. Ahmed, J. Mol. Liq. , 2017, 236 385-394.

51. Q.-X. Luo, M. Ji, M.-H. Lu, C. Hao, J.-S. Qiu and Y.-Q. Li, J. Mater. Chem. A, 2013, 1, 6530-6534. 
52. L. Peng, J. Zhang, S. Yang, B. Han, X. Sang, C. Liu and G. Yang, Green Chem., 2015, 17, 41784182.

53. A. Paul, A. P. C. Ribeiro, A. Karmakar, M. F. C. Guedes da Silva and A. J. L. Pombeiro, Dalton Trans., 2016, 12779-12789.

54. Y.-H. Hu, J.-C. Wang, S. Yang, Y.-A. Li and Y.-B. Dong, Inorg. Chem., 2017, DOI: 10.1021/acs.inorgchem.1027b01025.

55. D. Ma, B. Li, K. Liu, X. Zhang, W. Zou, Y. Yang, G. Li, Z. Shi and S. Feng, J. Mater. Chem. A, 2015, 3, 23136-23142.

56. L.-G. Ding, B.-J. Yao, W.-L. Jiang, J.-T. Li, Q.-J. Fu, Y.-A. Li, Z.-H. Liu, J.-P. Ma and Y.-B. Dong, Inorg. Chem., 2017, 56, 2337-2344.

57. J. Tharun, K.-M. Bhin, R. Roshan, D. W. Kim, A. C. Kathalikkattil, R. Babu, H. Y. Ahn, Y. S. Won and D.-W. Park, Green Chem., 2016, 18, 2479-2487.

58. B. Y. Park, K. Y. Ryu, J. H. Park and S. G. Lee, Green Chem., 2009, 11, 946-948.

59. Y. Ogasawara, S. Uchida, K. Yamaguchi and N. Mizuno, Chem.-Eur. J., 2009, 15, 4343-4349.

60. M. North, D. L. Usanov and C. Young, Chem. Rev., 2008, 108, 5146-5226.

61. J.-M. Brunel and I. P. Holmes, Angew. Chem., Int. Ed., 2004, 43, 2752-2778.

62. D. A. Evans, L. K. Truesdale and G. L. Carroll, J. Chem. Soc., Chem. Commun., 1973, 55-56.

63. R. J. H. Gregory, Chem. Rev., 1999, 99, 3649-3682.

64. V. Chechik, M. Conte, T. Dransfield, M. North and M. OmedesPujol, Chem. Commun., 2010, 46, 3372-3374.

65. Y. N. Belokon, M. North and T. Parsons, Org. Lett., 2000, 2, 1617-1619.

66. W. Xi, Y. Liu, Q. Xia, Z. Li and Y. Cui, Chem. - Eur. J., 2015, 21, 12581-12585.

67. D. Dang, P. Wu, C. He, Z. Xie and C. Duan, J. Am. Chem. Soc., 2010, 132, 14321-14323.

68. S. Horike, M. Dinca, K. Tamaki and J. R. Long, J. Am. Chem. Soc., 2008, 130, 5854-5855.

69. R. F. D'Vries, V. A. de la Peña-O'Shea, N. Snejko, M. Iglesias, E. Gutiérrez-Puebla and M. A. Monge, J. Am. Chem. Soc., 2013, 135, 5782-5792.

70. M. Gustafsson, A. Bartoszewicz, B. Martín-Matute, J. Sun, J. Grins, T. Zhao, Z. Li, G. Zhu and X. Zou, Chem. Mater., 2010, 22, 3316-3322.

71. G. Rajagopal, S. Selvaraj and K. Dhahagani, Tetrahedron: Asymmetry, 2010, 21, 2265-2270.

72. S. A. Pourmousavi and H. Salahshornia, Bull. Korean Chem. Soc., 2011, 32, 1575-1578.

73. R. El Osta, A. Carlin-Sinclair, N. Guillou, R. I. Walton, F. Vermoortele, M. Maes, D. de Vos and F. Millange, Chem. Mater., 2012, 24, 2781-2791.

74. M. Maes, F. Vermoortele, L. Alaerts, S. Couck, C. E. A. Kirschhock, J. F. M. Denayer and D. E. De Vos, J. Am. Chem. Soc., 2010, 132, 15277-15285.

75. A. Alizadeh and S. Rostamnia, Synthesis, 2010, 4057-4060.

76. K. S. Atwal, B. N. Swanson, S. E. Unger, D. M. Floyd, S. Moreland, A. Hedberg and B. C. Reilly, J. Med. Chem., 1991, 34, 806-811.

77. C. O. Kappe, Eur. J. Med. Chem., 2000, 35, 1043-1052.

78. R. A. Janis and D. J. Triggle, J. Med. Chem., 1983, 26, 775-785.

79. L. Martins, K. M. Vieria, L. M. Rios and D. Cardoso, Catal. Today, 2008, 133-135, 706-710.

80. C. M. McGuirk, M. J. Katz, C. L. Stern, A. A. Sarjeant, J. T. Hupp, O. K. Farha and C. A. Mirkin, J. Am. Chem. Soc., 2015, 137 919-925.

81. X. Zhang, Z. Zhang, J. Boissonnault and S. M. Cohen, Chem. Commun., 2016, 52 8585-8588.

82. R. Huisgen, in 1,3-Dipolar Cycloaddition Chemistry, ed. A. Padwa, Wiley, New York, 1984, p. 1.

83. V. V. Rostovtsev, L. G. Green, V. V. Fokin and K. B. Sharpless, Angew. Chem., Int. Ed., 2002, 41, 2596-2599.

84. C. Tornoe, C. Christensen and M. Meldal, J. Org. Chem., 2002, 67, 3057-3064.

85. S. K. Mamidyala and M. G. Finn, Chem. Soc. Rev., 2010, 39, 1252-1261.

86. Y. Hua and A. H. Flood, Chem. Soc. Rev., 2010, 39, 1262-1271.

87. K. D. Hanni and D. A. Leigh, Chem. Soc. Rev., 2010, 39, 1240-1251.

88. J. E. Hein and V. V. Fokin, Chem. Soc. Rev., 2010, 39, 1302-1315. 
89. M. Meldal and C. W. Tornøe, Chem. Rev., 2008, 108, 2952-3015.

90. S. Lober, P. Rodriguez-Loaiza and P. Gmeiner, Org. Lett., 2003, 5, 1753-1755.

91. J.-F. Lutz, Angew. Chem., Int. Ed., 2007, 46, 1018-1025.

92. R. Alvarez, S. Velazque, F. San, S. Aquaro, C. De, C. F. Perno, A. Karlsson, J. Balzarini and M. J. Camarasa, J. Med. Chem., 1994, 37, 4185-4194.

93. V. K. Y. Lo, Y. G. Liu, M. K. Wong and C. M. Che, Org. Lett. , 2006, 8, 1529-1532.

94. I. Matsuda, J. Sakakibara and H. Nagashima, Tetrahedron Lett. , 1991, 32 7431-7434.

95. C. P. Sar, T. Kalai, J. Jeko and K. Hideg, Arkivoc, 2012, 47-59.

96. Y. Himeda, N. Onozawa-Komatsuzaki, H. Sugihara and K. Kasuga, J. Am. Chem. Soc., 2005, 127, 13118-13119.

97. D. C. Stoian, E. Taboada, J. Llorca, E. Molins, F. Medina and A. M. Segarra, Chem. Commun., 2013, 49, 5489-5491.

98. T. Jiang, X. Ma, Y. Zhou, S. Liang, J. Zhang and B. Han, Green Chem., 2008, 10, 465-469.

99. W. Wang, S. Wang, X. Ma and J. Gong, Chem. Soc. Rev., 2011, 40, 3703-3727.

100. X. B. Lu and D. J. Darensbourg, Chem. Soc. Rev., 2012, 41, 1462-1484.

101. M. North, R. Pasquale and C. Young, Green Chem., 2010, 12, 1514-1539.

102. Q. He, J. W. O'Brien, K. A. Kitselman, L. E. Tompkins, G. C. T. Curtis and F. M. Kerton, Catal. Sci. Technol., 2014, 4, 1513-1528.

103. Y. J. Kim and R. S. Varma, J. Org. Chem., 2005, 70, 7882-7891.

104. V. Calo, A. Nacci, A. Monopoli, A. Monopoli and A. Fanizzi, Org. Lett., 2002, 4, 2561-2563.

105. Y. Xie, T. T. Wang, R. X. Yang, N. Y. Huang, K. Zou and W. Q. Deng, ChemSusChem, 2014, 7, 2110-2114.

106. C. G. Li, L. Xu, P. Wu, H. Wu and M. He, Chem. Commun., 2014, 50, 15764-15767.

107. Y. Zhang, S. Yin, S. Luo and C. T. Au, Ind. Eng. Chem. Res., 2012, 51, 3951-3957.

108. J. Q. Wang, D. L. Long, J. Y. Chen, F. Cai and L. N. He, J. Mol. Catal. A: Chem., 2006, 249, 143148.

109. W. Kleist, F. Jutx, M. Maciejewski and A. Baiker, Eur. J. Inorg. Chem., 2009, 3552-3561.

110. T. Yano, H. Matsui, T. Koike, H. Ishiguro, H. Fujihara, M. Yoshihara and T. Maeshima, Chem. Commun., 1997, 1129-1130.

111. H. Yasuda, L.-N. He and T. Sakakura, J. Catal. , 2002, 209 547-550.

112. Y. B. Xiong, H. Wang, R. M. Wang, Y. F. Yan, B. Zheng and Y. P. Wang, Chem. Commun., 2010, 46 3399-3401.

113. W. L. Dai, L. Chen, S. F. Yin, W. H. Li, Y. Y. Zhang, S. L. Luo and C. T. Au, Catal. Lett., 2010, 137 74-80.

114. C. R. Qi, J. W. Ye, W. Zeng and H. F. Jiang, Adv. Synth. Catal., 2010, 352 1925-1933.

115. T. Welton, Chem. Rev., 1999, 99 2071-2084.

116. E. R. Parnham and R. E. Morris, Acc. Chem. Res. , 2007, 40 1005-1013.

117. L. A. Blanchard, D. Hancu, E. J. Beckman and J. F. Brennecke, Nature 1999, 399 28-29.

118. S. Luo, X. Mi, L. Zhang, S. Liu, H. Xu and J.-P. Cheng, Angew. Chem. Int. Ed., 2006, 45 30933097.

119. K. Erfurt, I. Wandzik, K. Walczak, K. Matuszek and A. Chrobok, Green Chem., 2014, 163508 3514.

120. W. Wu, B. Han, H. Gao, Z. Liu, T. Jiang and J. Huang, Angew. Chem. Int. Ed. , 2004, 43 24152417.

121. M. Armand, F. Endres, D. R. MacFarlane, H. Ohno and B. Scrosati, Nat. Mater. , 2009, 8, 621629.

122. J. H. Clark and S. J. Tavener, Org. Proc. Res. Develop. , 2007, 11, 149-155.

123. R. V. Hangarge, D. V. Jarikote and M. S. Shingare, Green Chem. , 2002, 4, 266-268.

124. D.-Z. Xu, Y. Liu, S. Shi and Y. Wang, Green Chem. , 2010, 12 514-517.

125. A. Dhakshinamoorthy, M. Alvaro, P. Concepcion and H. Garcia, Catal. Commun. , 2011, 12 1018-1021. 
126. K. Schlichte, T. Kratzke and S. Kaskel, Micropor. Mesopor. Mater., 2004, 73 81-88.

127. D. Astruc, F. Lu and J. R. Aranzaes, Angew. Chem. Int. Ed. , 2005, 44, 7852-7872.

128. T. K. Shing, Y.-Y. Yeung and P. L. Su, Org. Lett., 2006, 8, 3149-3151.

129. D. H. Barton, H. Patin and F. Launay, New J. Chem., 1998, 22, 559-563.

130. J. Lee, O. K. Farha, J. Roberts, K. A. Scheidt, S. T. Nguyen and J. T. Hupp, Chem. Soc. Rev., 2009, 38, 1450-1459.

131. P. Yin, L. Chen, Z. Wang, R. J. Qu, X. G. Liu, Q. Xu and S. H. Ren, Fuel, 2012, 102, 499-505.

132. C. F. Oliveira, L. M. Dezaneti, F. A. C. Garcia, J. L. D. Macedo, J. A. Dias, S. C. L. Dias and K. S. P. Alvim, Appl. Catal., A 2010, 372, 153-161.

133. A. H. M. Fauzi, N. A. S. Amin and R. Mat, Appl. Energy, 2014, 114, 809-818.

134. C. Chen, Z. W. Wu, Y. G. Que, B. X. Li, Q. R. Guo, Z. Li, L. Wang, H. Wan and G. F. Guan, RSC Adv., 2016, 6, 54119-54128.

135. A. H. M. Fauzi and N. A. S. Amin, Energy Convers. Manage., 2013, 76, 818-827.

136. D. Lu, J. W. Zhao, Y. Leng, P. P. Jiang and C. J. Zhang, Catal. Commun., 2016, 83, 27-30.

137. L. Zhang, Y. Cui, C. Zhang, L. Wang, H. Wan and G. Guan, Ind. Eng. Chem. Res., 2012, 51, 1659016596.

138. Y. Jiang, J. Lu, K. Sun, L. Ma and J. Ding, Energy Convers. Manage., 2013, 76, 980-985.

139. Q. Wu, H. Wan, H. Li, H. Song and T. Chu, Catal. Today 2013, 200, 74-79.

140. A. H. Mohammad Fauzi and A. N. A. Saidina, Energy Convers. Manage., 2013, 76, 818 - 827.

141. B. Zhen, H. Li, Q. Jiao, Y. Li, Q. Wu and Y. Zhang, Ind. Eng. Chem. Res., 2012, 51, 10374-10380.

142. H. Zhang, F. Xu, X. Zhou, G. Zhang and C. Wang, Green Chem. , 2007, 9, 1208-1211.

143. M. C. Ferreira, A. J. Meirelles and E. A. Batista, Ind. Eng. Chem. Res. , 2013, 52, 2336-2351.

144. R. Chinchilla and C. Nájera, Chem. Rev., 2014, 114, 1783-1826.

145. L. Sun, J. Li, J. Park and H.-C. Zhou, J. Am. Chem. Soc., 2012, 134, 126-129.

146. D. Deng, Y. Yang, Y. Gong, Y. Li, X. Xu and Y. Wang, Green Chem., 2013, 15, 2525-2531.

147. Y. Yabe, T. Yamada, S. Nagata, Y. Sawama, Y. Monguchi and H. Sajiki, Adv. Synth. Catal., 2012, 354, 1264-1268.

148. W. Long, N. A. Brunelli, S. A. Didas, E. W. Ping and C. W. Jones, ACS Catal., 2013, 3, 1700-1708.

149. H. Sajiki, S. Mori, T. Ohkubo, T. Ikawa, A. Kume, T. Maegawa and Y. Monguchi, Chem. Eur. J., 2008, 14, 5109-5111.

150. H. Li, M. O'Keeffe, O. M. Yaghi and M. Eddaoudi, Nature, 1999, 402, 276-279.

151. M. Eddaoudi, J. Kim, N. Rosi, D. Vodak, J. Wachter, M. O'Keeffe and O. M. Yaghi, Science, 2002, 295, 469-472.

152. L. Alaerts, E. Seguin, H. Poelman, F. Thibault-Starzyk, P. A. Jacobs and D. E. De Vos, Chem.-Eur. J., 2006, 12, 7353-7363.

153. Y. K. Hwang, D. Y. Hong, J. S. Chang, S. H. Jhung, Y. K. Seo, J. Kim, A. Vimont, M. Daturi, C. Serre and G. Ferey, Angew. Chem., Int. Ed., 2008, 47, 4144-4148.

154. S. Gadipelli, J. Ford, W. Zhou, H. Wu, T. J. Udovicn and T. Yildirim, Chem.-Eur. J., 2011, 17, 6043-6047.

155. H. Furukawa, U. Müller and O. M. Yaghi, Angew. Chem., Int. Ed., 2015, 54, 3417-3430.

156. S. Yuan, L. Zou, J.-S. Qin, J. Li, L. Huang, L. Feng, X. Wang, M. Bosch, A. Alsalme, T. Cagin and H.-C. Zhou, Nature Commun., 2017, 8, 15356-15365.

157. D. Kim, D. W. Kim, O. Buyukcakir, M.-K. Kim, K. Polychronopoulou and A. Coskun, Adv. Funct. Mater., 2017, 27, DOI: adfm.201700706.

158. C. M. Miralda, E. E. Macias, M. Q. Zhu, P. Ratnasamy and M. A. Carreon, ACS Catal., 2012, 2, 180-183.

159. B. Bueken, N. V. Velthoven, T. Willhammar, T. Stassin, I. Stassen, D. A. Keen, G. V. Baron, J. F. M. Denayer, R. Ameloot, S. Bals, D. D. Vos and T. D. Bennett, Chem. Sci., 2017, 8, 3939-3948.

160. Q.-Q. Dang, Y.-F. Zhan, L.-N. Duan and X.-M. Zhang, Dalton Trans., 2015, 20027-20031.

161. Y. Zhu, Y. M. Wang, S. Y. Zhao, P. Liu, C. Wei, Y. L. Wu, C. K. Xia and J. M. Xie, Inorg. Chem., 2014, 53, 7692-7699. 
162. J. Yang, X. Wang, F. Dai, L. Zhang, R. Wang and D. Sun, Inorg. Chem., 2014, 53, 10649-10653.

163. R. Wang, Z. Wang, Y. Xu, F. Dai, L. Zhang and D. Sun, Inorg. Chem., 2014, 53, 7086-7088.

164. M. Fujita, Y. J. Kwon, S. Washizu and K. Ogura, J. Am. Chem. Soc., 1994, 116, 1151-1152.

165. R. F. D'Vries, M. Iglesias, N. Snejko, E. Gutierrez-Puebla and M. A. Monge, Inorg. Chem., 2012, 51, 11349-11355.

166. Y. Chen, X. Huang, S. Zhang, S. Li, S. Cao, X. Pei, J. Zhou, X. Feng and B. Wang, J. Am. Chem. Soc. , 2016, 138, 10810-10813.

167. T. Zhang, W. Liu, G. Meng, Q. Yang, X. Sun and J. Liu, ChemCatChem, 2017, 9, 1771-1775.

168. Z. Hu, Y. Peng, Y. Gao, Y. Qian, S. Ying, D. Yuan, S. Horike, N. Ogiwara, R. Babarao, Y. Wang, N. Yan and D. Zhao, Chem. Mater., 2016, 28, 2659-2667. 